\title{
Contrasting dynamics of electrons and protons in the near-Earth plasma sheet during dipolarization
}

\author{
Andrey Y. Malykhin ${ }^{1}$, Elena E. Grigorenko ${ }^{1,2}$, Elena A. Kronberg ${ }^{3,4}$, Rositza Koleva $^{5}$, Natalia Y. Ganushkina ${ }^{6,7}$, \\ Ludmila Kozak $^{8,9}$, and Patrick W. Daly ${ }^{3}$ \\ ${ }^{1}$ Space Research Institute of RAS, Moscow, Russia \\ ${ }^{2}$ Moscow Institute of Physics and Technology, Moscow, Russia \\ ${ }^{3}$ Max Planck Institute for Solar System Research, Göttingen, Germany \\ ${ }^{4}$ Ludwig Maximilian University of Munich, Munich, Germany \\ ${ }^{5}$ Space Research and Technologies Institute of BAS, Sofia, Bulgaria \\ ${ }^{6}$ Finnish Meteorological Institute, Helsinki, Finland \\ ${ }^{7}$ University of Michigan, Ann Arbor, MI, USA \\ ${ }^{8}$ Kyiv Taras Shevchenko University, Kiev, Ukraine \\ ${ }^{9}$ Space Research Institute National Academy of Sciences of Ukraine and State Space Agency of Ukraine, Kiev, Ukraine
}

Correspondence: Andrey Y. Malykhin (anmaurdreg@gmail.com)

Received: 13 December 2017 - Revised: 7 March 2018 - Accepted: 17 April 2018 - Published: 17 May 2018

\begin{abstract}
The fortunate location of Cluster and the THEMIS P3 probe in the near-Earth plasma sheet (PS) (at $X \sim-7-$ $-9 R_{\mathrm{E}}$ ) allowed for the multipoint analysis of properties and spectra of electron and proton injections. The injections were observed during dipolarization and substorm current wedge formation associated with braking of multiple bursty bulk flows (BBFs). In the course of dipolarization, a gradual growth of the $B_{Z}$ magnetic field lasted $\sim 13 \mathrm{~min}$ and it was comprised of several $B_{Z}$ pulses or dipolarization fronts (DFs) with duration $\leq 1 \mathrm{~min}$. Multipoint observations have shown that the beginning of the increase in suprathermal $(>50 \mathrm{keV})$ electron fluxes - the injection boundary - was observed in the PS simultaneously with the dipolarization onset and it propagated dawnward along with the onset-related DF. The subsequent dynamics of the energetic electron flux was similar to the dynamics of the magnetic field during the dipolarization. Namely, a gradual linear growth of the electron flux occurred simultaneously with the gradual growth of the $B_{Z}$ field, and it was comprised of multiple short $(\sim$ few minutes) electron injections associated with the $B_{Z}$ pulses. This behavior can be explained by the combined action of local betatron acceleration at the $B_{Z}$ pulses and subsequent gradient drifts of electrons in the flux pile up region through the numerous braking and diverting DFs. The nonadiabatic features occasionally observed in the electron spectra dur-
\end{abstract}

ing the injections can be due to the electron interactions with high-frequency electromagnetic or electrostatic fluctuations transiently observed in the course of dipolarization.

On the contrary, proton injections were detected only in the vicinity of the strongest $B_{Z}$ pulses. The front thickness of these pulses was less than a gyroradius of thermal protons that ensured the nonadiabatic acceleration of protons. Indeed, during the injections in the energy spectra of protons the pronounced bulge was clearly observed in a finite energy range $\sim 70-90 \mathrm{keV}$. This feature can be explained by the nonadiabatic resonant acceleration of protons by the bursts of the dawn-dusk electric field associated with the $B_{Z}$ pulses.

Keywords. Magnetospheric physics (Magnetotail; plasma sheet) - Space plasma physics (Transport processes)

\section{Introduction}

Rapid increase in the northward component of the Earth's magnetotail magnetic field $\left(B_{Z}\right)$, called dipolarization, is one of the key processes in tail dynamics. Spacecraft observations have shown that rapid enhancements in the $B_{Z}$ field represent spatial structures - dipolarization fronts (DFs) - which are typically observed at the leading edge of the earthwardmoving bursty bulk flows (BBFs) (e.g., Angelopoulos et al., 
1992; Nakamura et al., 2002; Runov et al., 2009). DFs are associated with electron and ion acceleration (e.g., Apatenkov et al., 2007; Asano et al., 2010; Zhou et al., 2010; Fu et al., 2011; Birn et al., 2011; Grigorenko et al., 2017) as well as with various wave activities, e.g., whistler emissions, lower hybrid and electron cyclotron waves (e.g., Le Contel et al., 2009; Zhou et al., 2009; Deng et al., 2010; Khotyaintsev et al., 2011; Hwang et al., 2011; Vilberg et al., 2014; Grigorenko et al., 2016). BBFs with embedded DFs transport energy and mass from a remote tail source to the near-Earth plasma sheet (PS), where the high-speed flows are slowed down and diverted (e.g., Sergeev et al., 2009; Ge et al., 2011 and references therein). This so-called flow braking region is located in the magnetotail approximately at $X \sim-10 R_{\mathrm{E}}$ (e.g., Shiokawa et al., 1997).

The energy transported by BBFs dissipates in the flow braking region through various channels including the adiabatic and nonadiabatic energization of particles (e.g., Khotyaintsev et al., 2011; Fu et al., 2011; Birn et al., 2013; Runov et al., 2015; Grigorenko et al., 2016; Khotyaintsev et al., 2017), the generation of compressional oscillations (e.g., Runov et al., 2014), the propagation of Alfvén waves out of the flow braking region into the auroral region (e.g., Ergun et al., 2015; Stawarz et al., 2015) and the generation of wave activity in a wide frequency range. The last may lead to additional energization and/or particles scattering and losses via processes of wave-particle interactions (e.g., Le Contel et al., 2009; Zhou et al., 2009; Deng et al., 2010; Khotyaintsev et al., 2011; Panov et al., 2013; Fu et al., 2014; Vilberg et al., 2014; Zhang and Angelopoulos, 2014; Grigorenko et al., 2016).

These phenomena have been intensively studied during recent decades. It was shown that dipolarizations occur at different timescales and can be roughly classified into two groups: (i) the propagating isolated DFs observed during a few minutes (e.g., Nakamura et al., 2002; Runov et al., 2009; Fu et al., 2011; Schmid et al., 2011) and (ii) the socalled "secondary" dipolarizations related to the flow braking and flux pile up in the near-Earth tail (e.g., Nakamura et al., 2009). The secondary dipolarizations are observed during much longer time periods (up to several hours) and usually are associated with the formation of the substorm current wedge (SCW) (McPherron et al., 1973; Sergeev et al., 2012; Yao et al., 2012). The magnetohydrodynamics (MHD) simulations performed by Birn and Hesse (2013) showed that the major SCW and pressure buildup occurred when the low-entropy flows are braked and the flows are diverted azimuthally in the near-Earth PS. The simulation results as well as multi-spacecraft observations showed that the braking flows are localized in space, while the associated increase in the $B_{Z}$ magnetic field (the dipolarization) evolves over a wider spatial range, spreading both azimuthally and radially (e.g., Nakamura et al., 2004; Birn and Hesse, 2013; Forsyth et al., 2014; Kronberg et al., 2017). This suggests that the
SCW consists of multiple small-scale "wedgelets" (e.g., Liu et al., 2013).

Sudden increases in fluxes of particles with energies of a few to hundreds of keVs - injections - are typical features of substorms observed in the near-Earth tail. Case and statistical studies showed a good correlation between injections and transient bursts of fast flow as well as earthward-propagating dipolarizing flux bundles (DFBs) (e.g., Sergeev et al., 2005; Apatenkov et al., 2008; Runov et al., 2009, 2011; Gabrielse et al., 2014). Injections are classified as "dispersionless" and "dispersed" (e.g., Sarris et al., 1976) based on the timing of the particle flux enhancements in different energy channels. If a spacecraft is located close to the injection source, the flux enhancement is observed simultaneously over a wide energy range, and such an injection is called dispersionless. If a spacecraft is distant from the source, there is an energydependent delay in the flux enhancement, with more energetic particles arriving first, due to energy-dependent gradient and curvature drifts, which result in a dispersed injection observation (e.g., Zaharia et al., 2000).

Injections have been intensively studied at a geosynchronous orbit (e.g., Lanzerotti et al., 1967; McIlwain, 1974; Mauk and McIlwain, 1974; Birn et al., 1997; Sarris et al., 2002). These studies considered injections as a result of the earthward motion of the "injection boundary", which represents a boundary between cold and hot plasma (e.g., Mauk and McIlwain, 1974). Another possibility is a compressionlike wave that heats and transports the plasma as it propagates from the tail to the inner magnetosphere (e.g., Moore et al., 1981).

Acceleration mechanisms responsible for energetic particle injections are studied using spacecraft observations, MHD simulations and test particle simulations. Birn (1987) traced particles in three-dimensional MHD simulations of dipolarization in the magnetotail and showed that particles are mainly accelerated by the betatron mechanism as they are transported by a time-dependent dawn-dusk electric field from the region of a weak magnetic field downtail to a stronger magnetic field at a geosynchronous orbit. Other simulations used an azimuthally wide earthward-propagating electromagnetic pulse to explain geosynchronous injections (e.g., Zaharia et al., 2000; Ganushkina et al., 2001, 2005, 2013; Sarris et al., 2002; Li et al., 2003). Authors concluded that injections can be caused by the earthward compression magnetic field perturbation and its associated electric field corresponding to a global magnetotail dipolarization.

Injections are observed not only at a geosynchronous orbit but also in the mid-tail region at $X \sim-9--30 R_{\mathrm{E}}$ simultaneously with DFs (e.g., Runov et al., 2009, 2011). It was shown that the betatron and Fermi mechanisms can be responsible for the increases in the suprathermal electron flux at and behind a DF (e.g., Asano et al., 2010; Ashour-Abdalla et al., 2011; Fu et al., 2011, 2012a; Birn et al., 2013). Gabrielse et al. (2016) demonstrated that a localized pulse of the inductive electric field associated with the earthward-propagating 
localized DFs or DFBs can accelerate electrons to suprathermal energies and, thus, be considered as an important source of short-lived ( $\leq 10 \mathrm{~min}$ ) injections. This study also showed that drift paths of energetic electrons are strongly influenced by the sharp magnetic gradients around a localized DFB. Birn et al. (2012) showed that not only adiabatic but also nonadiabatic acceleration processes related to the energization of resonant electrons due to the interaction with waves can operate in the course of dipolarization and flux pile up.

Magnetic dipolarizations are also responsible for the appearance of energetic ( $\geq 50 \mathrm{keV})$ ion fluxes in the near-Earth tail (e.g., Ipavich et al., 1984; Nosé et al., 2000; Ono et al., 2009; Keika et al., 2010; Luo et al., 2014; Kronberg et al., 2014, 2015; Grigorenko et al., 2017 and references therein). Among the proposed mechanisms, the nonadiabatic ion acceleration by the inductive electric field appearing in the course of the fast magnetic reconfiguration at a DF was considered in papers by Delcourt and Sauvaud (1994) and Delcourt (2002). Zhou et al. (2011) traced protons coming from the solar wind in the time-dependent magnetic and electric fields obtained from a global MHD simulation of a substorm. They explained the observation of dispersed ion injections by acceleration in two sources: (i) around the near-Earth $X$ line (at $X \sim-20 R_{\mathrm{E}}$ ), where particles are mostly accelerated nonadiabatically by strong electric fields (both inductive and potential), and (ii) in several localized regions between $X=-7 R_{\mathrm{E}}$ and $X=-18 R_{\mathrm{E}}$, where particles were accelerated in nonadiabatic motion under the potential electric field. The importance of nonadiabatic accelerations of protons in the course of their trapping by moving DFs was reported by e.g., Artemyev et al. (2012) and Ukhorskiy et al. (2013). Authors showed that both trapping and quasi-trapping can produce rapid acceleration of protons by more than an order of magnitude of their initial energies. Statistical analysis of Cluster observations at $X \geq-15 R_{\mathrm{E}}$ performed by Luo et al. (2014) confirmed that the increase in suprathermal ion population moving earthward is observed along with the high-speed bulk flows and DFs. Ono et al. (2009) demonstrated the importance of the nonadiabatic acceleration of protons and heavy ions in the course of their resonant interaction with the low-frequency magnetic fluctuations in the region behind the dipolarization front.

Generally, the adiabatic mechanisms of particle energization do not affect the spectral index $(\gamma)$ (e.g., Pan et al., 2012). Variations in the $\gamma$ value can be either due to the energy dispersion in particle injections or due to the nonadiabatic mechanisms of selective (resonant) acceleration and/or losses of a particular particle population in velocity distribution function. A comprehensive analysis of injections' properties and their spectra, plasma and fields' characteristics observed in a potential acceleration source - magnetic dipolarization - helps to understand mechanisms of particle acceleration and transport.

In the present paper we study the properties and spectra of electron and proton injections observed in the course of mag- netic dipolarization and SCW formation by using the fortunate location of Cluster spacecraft and the THEMIS P3 probe in the near-Earth PS. We discuss the characteristics of electron and proton injections associated with the braking and diversion of multiple BBFs and suggest possible mechanisms of particle acceleration. In Sect. 2 we described the data used; in Sect. 3 we presented an overview of the dipolarization event, its magnetic structure and evolution, as well as the related ground magnetic activity. In Sects. 3 and 4 we analyzed properties and energy spectra of injections of suprathermal electrons and protons observed in the course of dipolarization, respectively. We discussed the results of observations and possible mechanisms responsible for particle acceleration in Sect. 6 and made our conclusions in Sect. 7.

\section{Data description}

The magnetic field observations at the Cluster location are from the fluxgate magnetometers (FGMs) (spin- and highresolution $(22.4 \mathrm{~Hz})$ modes are used) (Balogh et al., 2001) operating on board four spacecraft. Ion moments are taken from the COmposition DIstribution Function (CODIF) instrument on board Cluster 4 (C4) (Réme et al., 2001). CODIF measures proton fluxes in the energy range of $0-38 \mathrm{keV}$. Fluxes of energetic electrons are measured by the Research with Adaptive Particle Imaging Detectors (RAPID) spectrometers on board four Cluster in the energy range of 20$400 \mathrm{keV}$ (Wilken et al., 2001). For the dipolarization event analyzed in this paper, the fluxes of energetic protons (40$1500 \mathrm{keV}$ ) were measured without gaps in the data only by the RAPID instrument on board C4. The electric field data are taken from the Electric Field and Wave (EFW) instrument (Gustafsson et al., 2001) on board four Cluster spacecraft. The electric and magnetic field wave spectra measured from 8 to $4096 \mathrm{~Hz}$ are provided by the Spatio-Temporal Analysis of Field Fluctuations (STAFF) experiment on board four Cluster spacecraft (Cornilleau-Wehrlin et al., 1997).

At the location of the THEMIS P3 probe, the ion and electron moments were calculated by using the reduced- and burst-mode observations from both the electrostatic analyzer (ESA) particle spectrometer, which measures plasma over the energy range from a few eV up to $30 \mathrm{keV}$ for electrons and up to $25 \mathrm{keV}$ for ions (McFadden et al., 2008), and the Solid State Telescope (SST) energetic particle spectrometer, which measures electrons and ions in the energy range of 25$7000 \mathrm{keV}$ (Angelopoulos, 2008). The magnetic and electric field data are taken from the magnetometer (FGM) (Auster et al., 2008) and from the Electric Field Instrument (EFI) (Bonnell et al., 2008), respectively. The electric and magnetic field wave spectra were obtained from the EFI and the Search Coil Magnetometer (SCM) (Roux et al., 2008) with frequencies measured from 1 to $4 \mathrm{kHz}$. If not specially mentioned, we use the geocentric solar magnetospheric (GSM) coordinate system everywhere in the paper. 

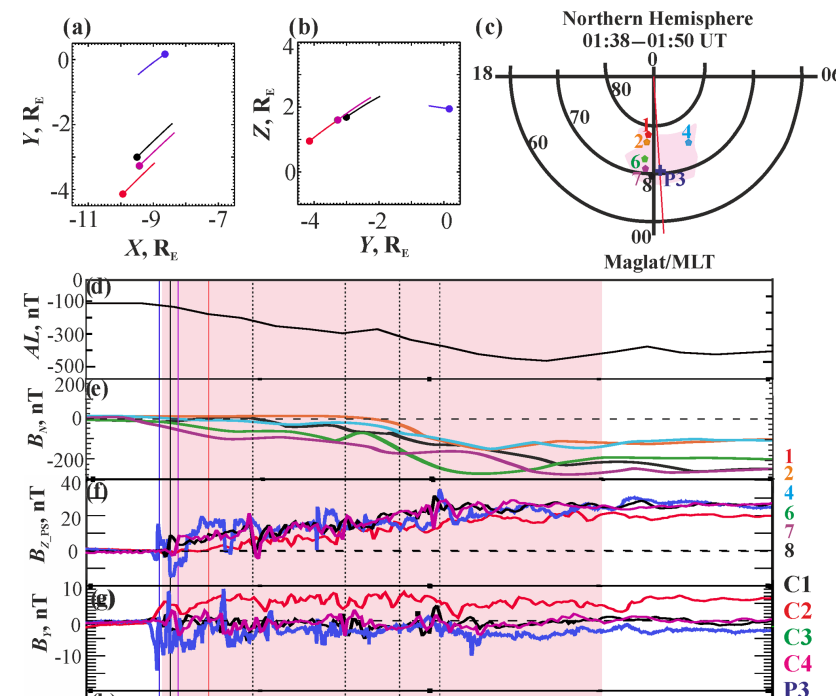

Maglat/MLT

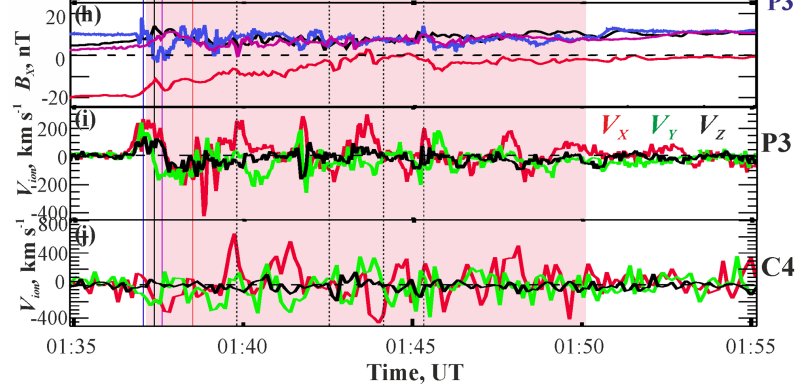

Figure 1. An overview of the dipolarization event observed on 20 July 2013. Panels (a) and (b) show the positions of the Cluster spacecraft and the THEMIS P3 probe in the near-Earth PS during the interval of interest in the $(X Y)_{\mathrm{GSM}}$ and $(X Z)_{\mathrm{GSM}}$ planes. Figure 1c shows the ionospheric footpoint of the P3 probe (marked by the blue crest) and the locations of the nearest ground magnetic stations indicated by the colored dots with the corresponding numbers (for the description of stations, see Table 1). The region of ground magnetic perturbation is shaded pink. Panels (d) and (e) display the time profiles of the AL index and the ground magnetic field component $B_{\mathrm{N}}$ measured by the stations located near the P3 probe ionospheric footpoint respectively. Panels $(\mathbf{f}-\mathbf{h})$ show three GSM components of the PS magnetic field ( $B_{Z_{-} \mathrm{PS}}, B_{Y_{-} \mathrm{PS}}$ and $B_{X_{-} \mathrm{PS}}$ ) observed by Cluster and the P3 probe. Panels (i) and (j) display three components of ion bulk velocity observed by the P3 probe and $\mathrm{C} 4$ respectively. The moments of onset-related DF observed by the $\mathrm{P} 3$ probe and $\mathrm{C} 1, \mathrm{C} 4$ and $\mathrm{C} 2$ are shown by solid vertical lines of blue, black, magenta and red color, respectively. The interval of dipolarization growth is shaded pink. Vertical dotted lines mark the $B_{Z}$ pulses for which the estimation of spatial scales was made (see explanations in Sect. 6).
Table 1. The magnetic coordinates of the $\mathrm{P} 3$ probe ionospheric footpoint and the nearest ground stations.

\begin{tabular}{lllrr}
\hline Nr & IAGA code & Name & Magnetic lat. & MLT \\
\hline & & P3 probe & 71.59 & 0.25 \\
1. & GHD & Godhavn & 77.99 & 23.69 \\
2. & ATU & Attu & 76.05 & 23.56 \\
3. & STF & Kangerlussuaq & 75.78 & 23.79 \\
4. & SCO & Ittoqqortoormiit & 74.43 & 1.86 \\
5. & SKT & Maniitsoq & 74.04 & 23.51 \\
6. & GHB & Nuuk & 72.96 & 23.56 \\
7. & FHB & Paamiut & 70.81 & 23.64 \\
8. & NAQ & Narssarssuaq & 69.41 & 23.92 \\
\hline
\end{tabular}

\section{An overview of the dipolarization event observed on 20 July 2013 at 01:35-01:55 UT}

Figure 1 shows an overview of the dipolarization event observed on 20 July 2013 between 01:35 and 01:55 UT. Figure $1 \mathrm{a}$ and $\mathrm{b}$ display the positions of the Cluster spacecraft and the THEMIS P3 probe in the near-Earth tail during the interval of interest in the $(X Y)_{\mathrm{GSM}}$ and $(X Z)_{\mathrm{GSM}}$ planes. Figure 1c shows the ionospheric footpoint of the P3 probe (marked by the blue crest) and the locations of the nearest ground magnetic stations indicated by the colored dots with the corresponding numbers. The names of these ground stations along with their magnetic latitudes and magnetic local times (MLTs) are listed in Table 1. Figure 1d presents the time profile of the AL index. Figure 1e shows the time profiles of the ground magnetic field components $B_{\mathrm{N}}$ measured by the ground stations located near the ionospheric footpoint of the P3 probe. The numbers of stations are shown in the right of these panels. For the ground magnetic field observations the coordinate system "NZE" is used. In this system, the $N$ axis points to the North Pole (i.e., the $B_{\mathrm{N}}$ represents the horizontal component of the ground magnetic field), the $Z$ axis is perpendicular to the Earth's surface and directed downward and the $E$ axis supplements the right three and is directed eastward.

Figure 1f-h show three GSM components of the PS magnetic field observed in the near-Earth magnetotail by four Cluster spacecraft and the THEMIS P3 probe. Figure 1i and $\mathrm{j}$ display three components of ion bulk velocity observed in the PS by the eTHEMIS P3 probe and by $\mathrm{C} 4$ respectively.

The dipolarization starts around 01:37 UT. The onset manifests in the observation of a sharp positive $B_{Z}$ jump denoting the arrival of DF, first, to the location of the THEMIS P3 probe at $\sim 01: 37$ UT (this moment is marked by the blue solid vertical line in Fig. 1), then, it arrived at Cluster 1 (C1) at $\sim 01: 37: 20 \mathrm{UT}$ and, finally, it reached the location of $\mathrm{C} 4$ at $\sim 01: 37: 35$ UT (these moments are marked in Fig. 1 by black and magenta solid vertical lines, respectively). Because of a very small distance between Cluster $3(\mathrm{C} 3)$ and $\mathrm{C} 4$ satellites ( a few tens of $\mathrm{km}$ ), the observations by $\mathrm{C} 3$ are very simi- 


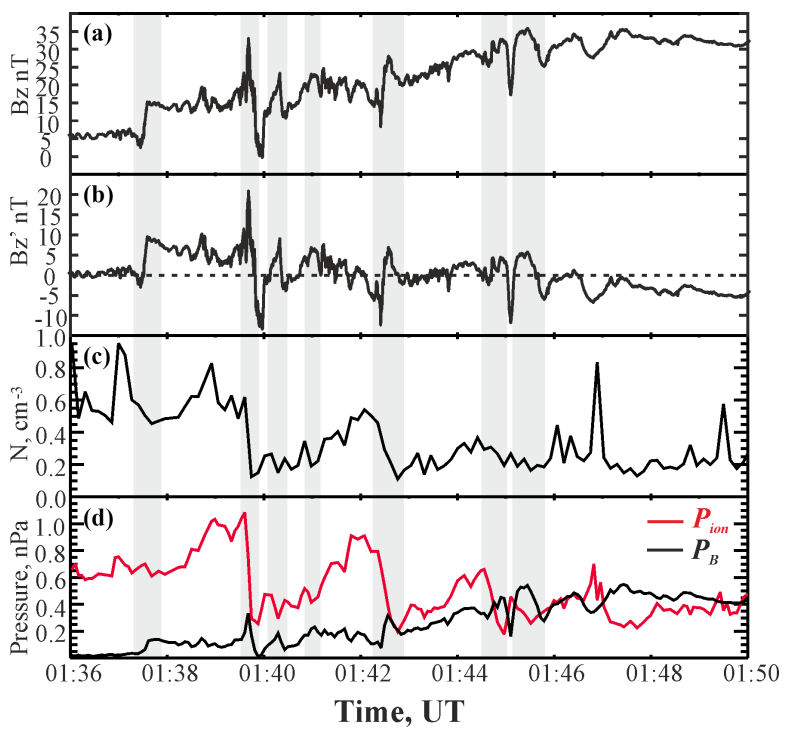

Figure 2. A zoom of the $B_{Z}$ (a) and $B_{Z}^{*}$ (b) fields, ion density (c), ion $\left(P_{\text {ion }}\right)$ and magnetic $\left(P_{\mathrm{B}}\right)$ pressure $(\mathbf{d})$ observed by $\mathrm{C} 4$ during the dipolarization growth. The intervals of strong $B_{Z}$ pulses are shaded grey.

lar to those obtained from $\mathrm{C} 4$ at this timescale, and they are not shown. The time delays between the observations of the onset-related DFs by different spacecraft indicate the dawnward propagation of the magnetic perturbation.

At Cluster $2(\mathrm{C} 2)$ the positive $B_{Z}$ field started to grow gradually a few minutes later than was observed by the other spacecraft (this moment is indicated by the red solid vertical line in Fig. 1). It is worth noting that $\mathrm{C} 2$ was located in the outer PS at the beginning of dipolarization growth (see Fig. 1h). This can be a cause why $\mathrm{C} 2$ did not record the onsetrelated DF.

After the onset, the gradual growth of the $B_{Z}$ component is observed by all spacecraft during $\sim 13$ min between $\sim 01: 37$ and 01:50 UT. The interval of dipolarization growth is shaded pink in Fig. 1. During the gradual growth of $B_{Z}$, numerous pulses of positive $B_{Z}$ field were observed. The $B_{Z}$ fluctuations could be related to MHD waves, which are often observed near DFs (e.g., Zhou et al., 2014). But as was reported by Zhou et al. (2014), the amplitude of these waves $\leq 2 \mathrm{nT}$. In our event the amplitude of strong $B_{Z}$ pulses exceeds $5 \mathrm{nT}$. Figure 2 displays a zoom of the $B_{Z}$ and $B_{Z}^{*}$ fields, ion density, plasma and magnetic pressure observed by $\mathrm{C} 4$ during the dipolarization growth. To obtain the time profile of $B_{Z}^{*}$ we subtracted the dipolarization growth $B_{Z}^{\prime}(t)$ from the observed $B_{Z}(t): B_{Z}^{*}(t)=B_{Z}(t)-B_{Z}{ }^{\prime}(t)$. The dipolarization growth $B_{Z}{ }^{\prime}(t)$ was approximated by the linear function at a large timescale (at 01:37:31-01:47:29 UT): $B_{Z}{ }^{\prime}(t)$ $=B_{Z 0}+k t$, where $B_{Z 0}=5.5$. nT is the value of the unperturbed $B_{Z}$ field averaged for $1 \mathrm{~min}$ before the dipolarization onset (at 01:36:30-01:37:30 UT); $k=0.0516 \mathrm{nT} \mathrm{s}^{-1}$ characterizes the speed of the gradual $B_{Z}$ growth. After the growth cessation at 01:47:29 $\mathrm{UT}$ the $B_{Z}^{*}(t)=$ constant $=36.4 \mathrm{nT}$. The intervals of the strongest $B_{Z}$ pulses with the amplitudes $\Delta B_{Z} \geq 5 \mathrm{nT}$ are shaded grey in Fig. 2. It is seen from the figure that the $B_{Z}$ pulses have the typical characteristics of DFs, such as the rapid increase of the $B_{Z}$ component preceded by the negative $B_{Z}$ variation, the decrease in density behind the front, and the decrease in plasma pressure, while magnetic pressure increases across the front (e.g., Shiokawa et al., 2005; Zhou et al., 2009; Runov et al., 2009). Grigorenko et al. (2018) studied the magnetic gradients observed by closely located $\mathrm{C} 3$ and $\mathrm{C} 4$ in the vicinity of the strongest $B_{Z}$ pulse detected by $\mathrm{C} 4$ at $\sim$ 01:39:41 UT. They have shown the presence of intense localized and approximately fieldaligned electric currents near the leading and trailing edges of the $B_{Z}$ pulse. These currents can be a part of a multiscale electric current system usually associated with DFs (e.g., Fu et al., 2012; Liu et al., 2013; Balikhin et al., 2014).

The exact moments of the first DF observation by different spacecraft (we will call this front the onset-related DF) are presented in Table 2, where this DF is marked as "DF0". Assuming 1-D magnetic structure of DF we calculate the normal $(N)$ to the DF0 by using minimum variance analysis (MVA) (Paschmann and Daly, 1998) applied to the highresolution magnetic field observations obtained by $\mathrm{P} 3, \mathrm{C} 1$ and $\mathrm{C} 4$ spacecraft. We found that at the $\mathrm{P} 3$ location the $N_{\mathrm{P} 3}=$ $[0.9,-0.2,-0.4]$, at the $\mathrm{C} 1$ location the $N_{\mathrm{C} 1}=[-0.6,-0.6$, $0.5]$ and at the $\mathrm{C} 3-\mathrm{C} 4$ location the MVA results are very similar and give $N_{\mathrm{C} 3, \mathrm{C} 4}=[-0.2,-0.8,0.5]$. At each spacecraft location the normal to the front was well defined: the ratios of the intermediate to minimum eigenvalues as well as the ratios of the maximum to intermediate eigenvalues exceeded 10.0. We estimated the propagation velocity of DF0 by using the magnetic field observations from $\mathrm{C} 3$ and $\mathrm{C} 4$, which are located close to each other. The resulting minimum variance directions obtained at $\mathrm{C} 3$ and $\mathrm{C} 4$ locations were very closely aligned to each other. The close alignment of the individual minimum and maximum variance directions allows us to treat the DF as a planar structure on spatial scales of the order of the satellite separation distances. Assuming that the motion of the leading edge of the DF0 structure is along its normal direction $N_{\mathrm{C} 3, \mathrm{C} 4}$, the time delay between $\mathrm{C} 3$ and $\mathrm{C} 4$ can be used to identify its velocity. We found that DF0 propagated mainly dawnward with $\left|V_{\text {prob }}\right| \sim 130 \mathrm{~km} \mathrm{~s}^{-1}$. This behavior is typical of the motion of DFs near the flow braking region (e.g., Ge et al., 2011; Birn and Hesse, 2013.

During this interval, 10 positive $B_{Z}$ pulses with amplitude $\geq 5 \mathrm{nT}$ were observed by $\mathrm{P} 3$ and $\mathrm{C} 1$ satellites; $7 B_{Z}$ pulses were recorded by $\mathrm{C} 4$ and $\mathrm{C} 3$ and only 3 pulses were observed by $C 2$ (see Fig. 1f). Thus, a number of $B_{Z}$ pulses was decreasing as the dipolarization propagated dawnward. The time moments of the $B_{Z}$ pulses (DFs) observed by the corresponding spacecraft as well as the directions of normals $N$ to their fronts are listed in Table 2.

Figure $1 \mathrm{i}$ and $\mathrm{j}$ show three components of ion bulk velocity measured by the ESA spectrometer on board the $\mathrm{P} 3$ probe 
Table 2. Time moments of the $B_{Z}$ pulses (DFs) and electron injections observed by the P3 probe and Cluster spacecraft during dipolarization.

\begin{tabular}{|c|c|c|c|c|}
\hline \multirow[t]{2}{*}{ Spacecraft } & \multicolumn{2}{|l|}{$B_{Z}$ pulses } & \multicolumn{2}{|l|}{ Injections } \\
\hline & Time moments & MVA normals & $\begin{array}{l}\text { Time moments for } \\
65.5 \mathrm{keV} \text { (P3) } \\
68.1 \mathrm{keV} \text { (Cluster) }\end{array}$ & $\begin{array}{l}\text { Time moments for } \\
139 \mathrm{keV}(\mathrm{P} 3) \\
127.5 \mathrm{keV} \text { (Cluster) }\end{array}$ \\
\hline P3 & $\begin{array}{l}\text { DF0: } 01: 37: 00.027 \\
\text { DF1: 01:38:16.875 } \\
\text { DF2: 01:39:27.576 } \\
\text { DF3: } 01: 42: 04.348 \\
\text { DF4: } 01: 43: 05.827 \\
\text { DF5: } 01: 45: 18.007 \\
\text { DF6: } 01: 47: 54.779\end{array}$ & $\begin{array}{l}{\left[\begin{array}{lll}0.9 & -0.2 & -0.4\end{array}\right]} \\
{\left[\begin{array}{lll}-0.8 & 0.4 & 0.45\end{array}\right]} \\
{\left[\begin{array}{lll}0.3 & 0.95 & -0.1\end{array}\right]} \\
{\left[\begin{array}{llll}0.6 & 0.7 & -0.4\end{array}\right]} \\
{\left[\begin{array}{llll}0.1 & 0.96 & -0.2\end{array}\right]}\end{array}$ & $\begin{array}{l}I_{\mathrm{e}} 1: 01: 38: 23.302 \\
I_{\mathrm{e}} 2: 01: 39: 24.781 \\
I_{\mathrm{e}} 3: 01: 42: 01.553 \\
I_{\mathrm{e}} 4: 01: 43: 12.254 \\
I_{\mathrm{e}} 5: 01: 45: 27.508 \\
I_{\mathrm{e}} 6: 01: 47: 48.910\end{array}$ & $\begin{array}{l}01: 38: 23.302 \\
01: 39: 24.781 \\
01: 42: 01.553 \\
01: 43: 18.402 \\
01: 45: 27.508 \\
01: 47: 58.132\end{array}$ \\
\hline $\mathrm{C} 1$ & $\begin{array}{l}\text { DF0: 01:37:20.871 } \\
\text { DF1: 01:39:39.933 } \\
\text { DF2: 01:40:15.880 } \\
\text { DF3: 01:40:22.571 } \\
\text { DF4: 01:42:23.749 } \\
\text { DF5: 01:44:43.168 } \\
\text { DF6: 01:45:07.876 } \\
\text { DF7: 01:46:38.413 }\end{array}$ & $\begin{array}{l}{\left[\begin{array}{llll}-0.6 & -0.6 & 0.5\end{array}\right]} \\
{\left[\begin{array}{lll}0.5 & 0.8 & -0.3\end{array}\right]} \\
{\left[\begin{array}{lll}0.8 & 0.6 & 0.0\end{array}\right]} \\
{\left[\begin{array}{llll}0.6 & 0.7 & -0.4\end{array}\right]} \\
{\left[\begin{array}{llll}0.8 & -0.6 & 0.0\end{array}\right]} \\
{\left[\begin{array}{llll}0.9 & 0.4 & 0.1\end{array}\right]} \\
{\left[\begin{array}{llll}0.96 & 0.06 & -0.3\end{array}\right]}\end{array}$ & $\begin{array}{l}I_{\mathrm{e}} 1: 01: 38: 37.766 \\
I_{\mathrm{e}} 2: 01: 39: 20.097 \\
I_{\mathrm{e}} 3: 01: 40: 53.223 \\
I_{\mathrm{e}} 4: 01: 42: 30.581 \\
I_{\mathrm{e}} 5: 01: 44: 20.638 \\
I_{\mathrm{e}} 6: 01: 45: 45.298 \\
I_{\mathrm{e}} 7: 01: 47: 09.956\end{array}$ & $\begin{array}{l}01: 38: 37.766 \\
01: 39: 20.097 \\
01: 40: 53.223 \\
01: 42: 26.348 \\
01: 44: 20.638 \\
01: 45: 45.298 \\
01: 46: 53.025\end{array}$ \\
\hline $\mathrm{C} 4$ & $\begin{array}{l}\text { DF0: 01:37:35.448 } \\
\text { DF1: 01:39:40.908 } \\
\text { DF2: 01:40:19.575 } \\
\text { DF3: 01:42:34.355 } \\
\text { DF4: 01:44:43.960 } \\
\text { DF5: 01:45:27.088 } \\
\text { DF6: 01:47:24.698 }\end{array}$ & $\begin{array}{l}{\left[\begin{array}{lll}-0.2 & -0.8 & 0.5\end{array}\right]} \\
{\left[\begin{array}{lll}0.9 & -0.3 & 0.1\end{array}\right]} \\
{\left[\begin{array}{lll}0.65 & 0.7 & 0.2\end{array}\right]} \\
{\left[\begin{array}{lll}0.95 & -0.2 & -0.3\end{array}\right]} \\
{\left[\begin{array}{lll}0.96 & 0.25 & -0.15\end{array}\right]} \\
{\left[\begin{array}{lll}0.7 & -0.6 & -0.25\end{array}\right]} \\
{\left[\begin{array}{lll}0.6 & 0.8 & -0.1\end{array}\right]}\end{array}$ & $\begin{array}{l}I_{\mathrm{e}} 1: 01: 38: 50.996 \\
I_{\mathrm{e}} 2: 01: 39: 36.343 \\
I_{\mathrm{e}} 3: 01: 41: 07.038 \\
I_{\mathrm{e}} 4: 01: 42: 29.487 \\
I_{\mathrm{e}} 5: 01: 44: 29.039 \\
I_{\mathrm{e}} 6: 01: 47: 38.674\end{array}$ & $\begin{array}{l}- \\
01: 39: 23.976 \\
01: 41: 07.038 \\
01: 42: 29.487 \\
01: 44: 24.918 \\
01: 47: 13.939\end{array}$ \\
\hline $\mathrm{C} 2$ & $\begin{array}{l}\text { DF1: 01:39:53.064 } \\
\text { DF2: 01:42:19.531 } \\
\text { DF3: 01:43:57.472 }\end{array}$ & $\begin{array}{l}{\left[\begin{array}{lll}0 & 0.99 & 0.15\end{array}\right]} \\
{\left[\begin{array}{lll}0.9 & -0.3-0.3\end{array}\right]} \\
{\left[\begin{array}{lll}0.8 & -0.4 & -0.3\end{array}\right]}\end{array}$ & $\begin{array}{l}I_{\mathrm{e}} 1: 01: 39: 48.479 \\
I_{\mathrm{e}} 2: 01: 42: 25.994 \\
I_{\mathrm{e}} 3: 01: 48: 34.913\end{array}$ & $\begin{array}{l}01: 39: 48.479 \\
01: 42: 25.994 \\
01: 46: 51.284\end{array}$ \\
\hline
\end{tabular}

and by the CODIF instrument on board $\mathrm{C} 4$, respectively. One can see that the dipolarization onset at the P3 location is associated with the arrival of the earthward BBF $\left(V_{X} \sim\right.$ $250 \mathrm{~km} \mathrm{~s}^{-1}$ ), while at the $\mathrm{C} 4$ location, the reflected tailward flow is observed at the time of the onset. This is in agreement with the assumption that the onset-related DF arrived at the Cluster spacecraft after its braking and deflection near the location of the P3 probe. However, later, at 01:39:40 UT, C4 observed a high-speed flow with $V_{X} \sim 600 \mathrm{~km} \mathrm{~s}^{-1}$ and with the embedded $B_{Z}$ pulse or DF (in Table 2 it is marked as DF1 for $\mathrm{C} 4$ data). This flow most likely arrived at the $\mathrm{C} 4$ location directly from a remote source located downtail. Then, after 01:42 UT, and until the end of the dipolarization growth, the flow oscillations were observed by both P3 and C4. The similar phenomenon was discussed earlier in detail by e.g., Panov et al. $(2010,2015)$ in the context of the oscillatory flow braking through alternating field-aligned currents.
The onset of dipolarization at P3 and C1-3,4 locations is observed almost simultaneously with the start of the negative variation of the horizontal component of the ground magnetic field, $B_{\mathrm{N}}$ (see Fig. 1e) and with the growth of the absolute value of the AL index (see Fig. 1d). These observations indicate the formation of the SCW (e.g., Birn and Hesse 2013). From Fig. 1e it is seen that the growth of negative $B_{\mathrm{N}}$ started first at Paamiut station (no. 7), i.e., a little duskward of the P3 ionospheric footpoint (see Fig. 1c and Table 1). Then, the ionospheric perturbation rapidly expands equatorward and poleward (the negative $B_{\mathrm{N}}$ variations started at stations no. 6 and no. 8), and, then, the perturbation expanded towards the post-midnight sector (to no. 4 station) and farther poleward (to no. 2 station). The evolution of the ground magnetic field perturbation and the dynamics of the dipolarization in the near-Earth PS demonstrate similar features. Namely, the timescale of dipolarization growth in the PS is similar to the timescale of the growth of the negative value of 
(a) $\mathbf{P 3}$

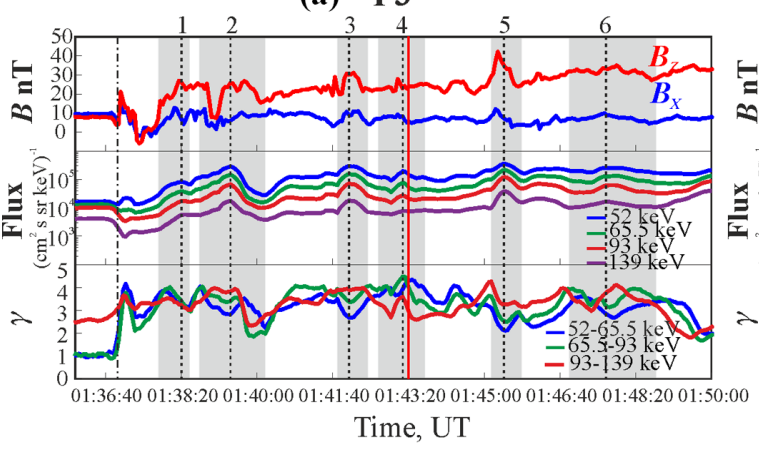

(c) $\mathrm{C} 4$

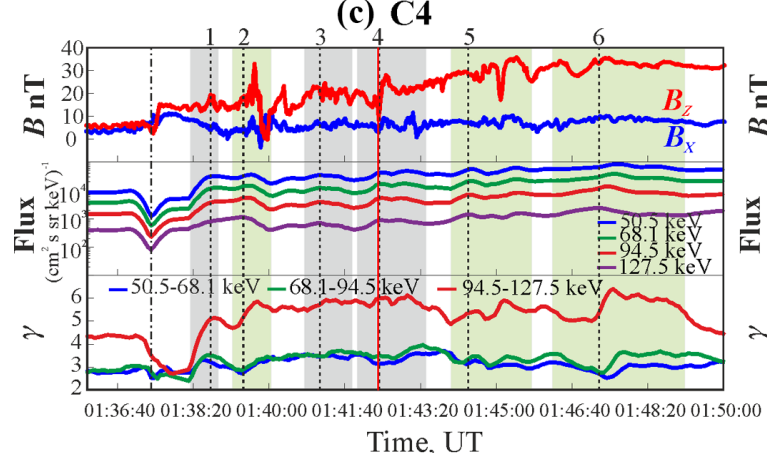

(b) $\mathrm{C} 1$

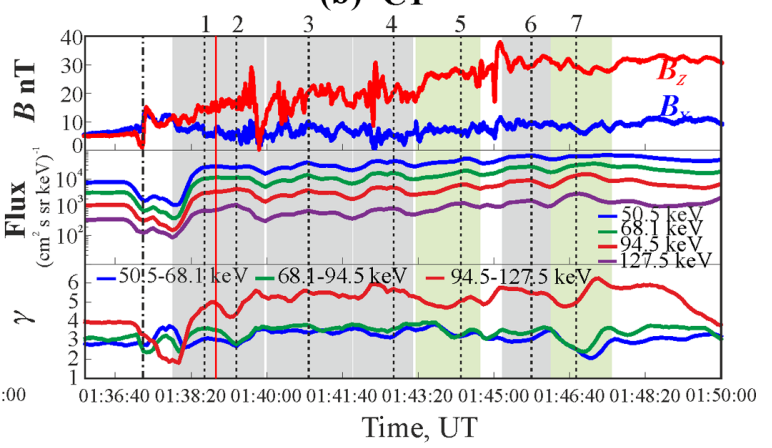

(d) $\mathrm{C} 2$

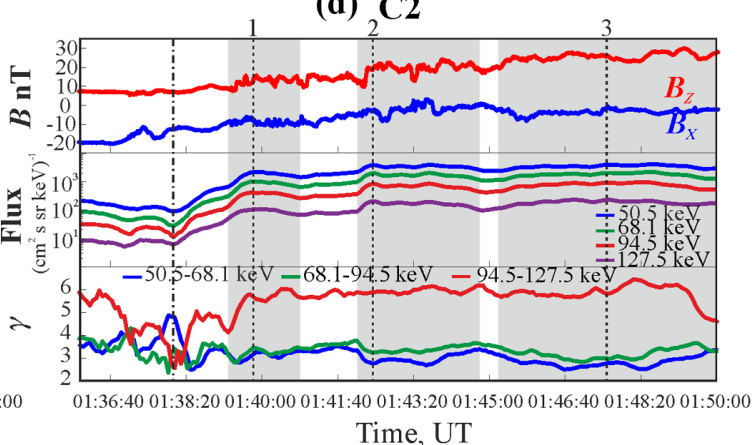

Figure 3. The dynamics of suprathermal electron fluxes and spectral index $\gamma$ observed by $\mathrm{P} 3, \mathrm{C} 1, \mathrm{C} 4$ and $\mathrm{C} 2$ satellites. In each plot the following data from a given spacecraft are shown from top to bottom: the time profiles of $B_{X}$ and $B_{Z}$ magnetic field components, the time profiles of electron fluxes in several energy ranges and the spectral index $\gamma$ calculated for three energy ranges indicated in the corresponding panel. The time moment of dipolarization onset at each spacecraft is shown by the vertical dashed and dotted line. The intervals of dispersed injections are shaded light green and the intervals of dispersionless injections are shaded grey. The moments of peak electron flux observations in each injection are indicated by vertical dashed lines. The moments of $\gamma$ variations which cannot be explained by the time-of-flight energy dispersion are indicated by red solid vertical lines.

the horizontal component of the ground magnetic field, $B_{\mathrm{N}}$. Also, both the ground magnetic perturbation and the dipolarization in the magnetotail propagate in the same direction, i.e., towards the post-midnight sector (or dawnward).

Thus, the observations presented in this section show that the dipolarization in the near-Earth PS is caused by the braking, diversion and oscillations of multiple BBFs. This results in a complicated magnetic field perturbation, which includes several pulses of the $B_{Z}$ field observed in the PS during the dipolarization growth. Since these pulses had the characteristics typical of DFs we may assume that they represent the DFs embedded into the plasma flows. In the following sections we discuss how this magnetic field perturbation affects the dynamics and spectra of suprathermal $(>50 \mathrm{keV})$ electron and proton fluxes observed in the PS during the dipolarization.

\section{Properties and spectra of electron injections observed in the course of dipolarization and flux pile up}

The onset of dipolarization was followed by the increase in flux of suprathermal $(>50 \mathrm{keV}$ ) electrons. To quantify the spectra of suprathermal electrons we assume that the differential flux of electrons $\left(J_{\mathrm{e}}\right)$ can be described by a power law in energy $(W): J_{\mathrm{e}} \sim W^{-\gamma}$ (e.g., Øieroset et al., 2002; Imada et al., 2007). In this case the spectral index $\gamma$ can be calculated according to Eq. (6) in a paper by Kronberg and Daly (2013):

$\gamma=\frac{\ln \left(J_{\mathrm{e} 2} / J_{\mathrm{e} 1}\right)}{\ln \left(E_{\mathrm{eff} 2} / E_{\mathrm{eff} 1}\right)}$.

Here, the $J_{\mathrm{e} 2}$ and $J_{\mathrm{e} 1}$ are the differential flux of electrons in the neighboring energy channels. We used the lowest energies of the corresponding channels as the effective energies $E_{\text {eff2 }}$ and $E_{\text {eff1 }}$.

In Fig. 3, we present four plots with observations obtained by the $\mathrm{P} 3$ probe and the $\mathrm{C} 1, \mathrm{C} 4$ and $\mathrm{C} 2$ spacecraft. Each plot contains from top to bottom: the $B_{Z}$ and $B_{X}$ magnetic field components (shown for reference in red and blue, respec- 
tively), electron fluxes at different energy channels and spectral index $\gamma$ calculated for the following pairs of energy channels: $50.5-68.1 \mathrm{keV}$ (blue line), 68.1-94.5 keV (green line) and $94.5-127.5 \mathrm{keV}$ (red line). For details of the $\gamma$ calculation method see Kronberg and Daly (2013).

The beginning of increase in energetic electron flux was observed by each spacecraft after the onset of dipolarization, i.e., after the first positive enhancement in the $B_{Z}$ field (at each plot in Fig. 3, the time moment of the dipolarization onset is shown by the black vertical dashed and dotted line). After the dipolarization onset, the behavior of the energetic electron fluxes resembles the dynamics of the $B_{Z}$ field in the PS. Namely, a gradual growth of the energetic electron flux is observed along with the gradual growth of $B_{Z}$ field at timescale $\sim 13 \mathrm{~min}$, i.e., between 01:38 and 01:50 UT.

During the interval of the flux growth and even after it, multiple and short ( $\sim$ a few minutes) flux enhancements are observed. We considered an enhancement in suprathermal electron fluxes as an "injection" if the fluxes in two and more energy channels increased more than 1.7 times. As a result, we identified six and seven injections at $\mathrm{P} 3$ and $\mathrm{C} 1$ locations, respectively, and six and three injections at $\mathrm{C} 4$ and $\mathrm{C} 2$ locations, respectively ( $\mathrm{C} 3$ observations are similar to $\mathrm{C} 4$ ones and are not shown). In Fig. 3, these injections are marked by numbers at the top of the corresponding plot. The dispersionless injections are shaded grey and dispersed injections are shaded light green. The exact time moments of the electron flux maximum observed by the $\mathrm{P} 3$ probe for 65.5 and $139 \mathrm{keV}$ and by the Cluster spacecraft for 68.1 and $127.5 \mathrm{keV}$ electrons in each injection are listed in Table 2 along with the time moments of the $B_{Z}$ pulses or DFs, which were detected during the dipolarization growth.

The first injection (marked as " $I_{\mathrm{e}} 1$ " in Table 2) of suprathermal electrons was observed by the $\mathrm{P} 3$ probe at 01:38:23 UT, i.e., at the beginning of the dipolarization growth just after the $B_{Z}$ pulse, marked as "DF1" for P3 data in Table 2. This injection was dispersionless, which indicates the close proximity of the spacecraft to the acceleration source. At the $\mathrm{C} 1$ location, the first injection was observed at 01:38:37 UT, i.e., $14 \mathrm{~s}$ later than at the P3 location and after the onset-related DF (DF0 in Table 2). This injection also was dispersionless. C4 observed the first injection even later than P3 and $\mathrm{C} 1$, at 01:38:50 UT, and also after the arrival of the onset-related DF0 at its location. Finally, C2 recorded the first injection at 01:39:48 UT, when the dipolarization had started at its location.

Thus, the time delays in observations of the first injection by different spacecraft indicate the dawnward propagation of the injection boundary, i.e. in the same direction as the propagation of dipolarization onset. At all spacecraft, the injection was observed behind the onset-related DF. The following injections detected during the period of gradual dipolarization growth, i.e., $I_{\mathrm{e}} 2-6$ at P3 and $\mathrm{C} 1$ locations and $I_{\mathrm{e}} 2-6$ at the $\mathrm{C} 4$ location, were also observed either at or between the corresponding $B_{Z}$ pulses (for the exact moments see Table 2).
The majority of dispersionless injections were observed during the first part of the interval of dipolarization growth, i.e., between 01:38 and 01:48 UT, when the strongest $B_{Z}$ pulses were detected. This shows that during this period, electron acceleration to suprathermal energies occurred very close to the corresponding satellite.

After the end of dipolarization growth, the injection(s) were also observed at all spacecraft locations, e.g., $I_{\mathrm{e}} 7$ at the $\mathrm{C} 1$ location and $I_{\mathrm{e}} 6$ at the $\mathrm{C} 4$ location. Contrary to the previous ones, these injections were dispersed (see Fig. 3). The dispersed character of electron injections can be caused by the gradient drifts from a remote source (e.g., Gabrielse et al., 2016). Thus, at the end of dipolarization growth, energetic electrons arrived at the spacecraft locations either from a remote acceleration source or as an "echo" of the previous injections. The echoes can represent the previously accelerated electron population, which experiences the bouncing oscillations along magnetic field lines.

To check the origin of the dispersed injection observed after the end of dipolarization growth, we roughly estimate a source location by using the time delays $(t)$ in observations of electron flux enhancements at the neighboring energy channels for injection $I_{\mathrm{e}} 6$ at $\mathrm{C} 4$. The distance to the source can be estimated as $\Delta R=\frac{V_{2} V_{1}}{V_{1}-V_{2}} \Delta t$ (where $V_{1}$ and $V_{2}$ are electron drifting velocities calculated for the lowest energies of two neighboring energy channels). For electron enhancements in the energy channels 127.5 and $94.5 \mathrm{keV}$, we obtained the distance to the source $R \sim 70 R_{\mathrm{E}}$, and for energy channels 68.1 and $50.5 \mathrm{keV}$ we obtained $R \sim 45 R_{\mathrm{E}}$. Thus, we may suggest that the $I_{\mathrm{e}} 6$ injection observed at the end of dipolarization may represent an echo of the previous injections.

During the dipolarization event the spectral index $\gamma$ of suprathermal electrons experienced variations as is seen from the bottom panels of each plot presented in Fig. 3. Generally, variations of $\gamma$ reflect the differences in energization and/or losses in a finite energy range of a particle spectrum. These differences can be caused by some nonadiabatic mechanisms, e.g., wave-particle interactions, which lead to the selective acceleration and/or scattering into the loss cone of some population in electron energy distribution. Another cause of $\gamma$ temporal variations is the time-of-flight effect due to the energy-dependent electron drifts from a remote source.

During the dipolarization event the pronounced $\gamma$ variations with amplitude $|\Delta \gamma|>1.0$ were mostly observed during the dispersed injections, and, thus, were caused by timeof-flight effects. In such cases the negative $\gamma$ variations were due to the first arrival of more energetic electrons, while the positive variations following were caused by the later arrival of lower energy electrons and simultaneous loss of more energetic electrons. This pattern is clearly observed for the dispersed injections 5 and 7 at the $\mathrm{C} 1$ location and 5 and 6 at the C4 location (see Fig. 3).

However, at some moments within the dispersed injections the behavior of $\gamma$ cannot be explained by the time-of-flight effects due to gradient drifts. Also, $\gamma$ variations observed dur- 
ing the dispersionless injections should be caused by other mechanisms. In Fig. 3 we indicate some of such moments by red vertical lines.

In the left part of Fig. 4 we show electron fluxes along with the wave data observed by $\mathrm{P} 3, \mathrm{C} 1$ and $\mathrm{C} 4$ within short time intervals around the moments marked by red vertical lines in Fig. 3 (no. 4 - for $\mathrm{P} 3$, no. 1 - for $\mathrm{C} 1$ and no. 4 - for $\mathrm{C} 4$ ). In the right part of Fig. 4 we plot the electron spectra observed by the corresponding spacecraft at the time moments indicated in the panels on the left by the vertical lines of the corresponding color. It is seen that for all intervals shown in Fig. 4 the bursts of high-frequency electrostatic or electromagnetic fluctuations with frequencies up to electron gyrofrequency, $f_{\text {ce }}$, are observed.

At the P3 location the flattening of the spectrum (indicated by a dashed bar) is observed just after the end of the bursts of electron cyclotron harmonic $(\mathrm{ECH})$ waves (see the left part of the "P3 no. 4" plot in Fig. 4). This causes a significant decrease in $\gamma(\Delta \gamma \sim 1.0)$ calculated for 65.5-93 and 93-139 keV energy ranges (see Fig. 3). By the end of the interval, the flattening of spectra expanded to the lower energy range (see the green spectrum at 01:43:30 UT). This spectral feature was also associated with the ECH bursts.

$\mathrm{C} 1$ spacecraft also observed the changes in the electron energy spectrum in a finite energy range during the dipolarization growth (see the interval "C1 no. 1" in Fig. 4). During this interval the most prominent flattening of spectra in the energy range of $68.1-94.5 \mathrm{keV}$ was observed at 01:38:20 01:39:20 UT (compare the red and magenta spectra on the right of the $\mathrm{C} 1$ no. 1 plot in Fig. 4). These spectra were detected just after the bursts of electromagnetic emissions in a wide frequency range from a few $\mathrm{Hz}$ and up to $\sim 100 \mathrm{~Hz}$ and after the ECH bursts.

Similar features were detected by $\mathrm{C} 4$ during injection no. 4 (see Fig. 4) around 01:44:41 UT. At this time the flattening of electron spectrum was observed in a wider energy range: $50.5-94.5 \mathrm{keV}$ and at the end of the burst of high-frequency electrostatic fluctuations (compare black and red spectra in the right part of "C4 no. 4" plot in Fig. 4).

Our observations, thus, show that the $\gamma$ variations, which were observed in a finite energy range and cannot be explained by the time-of-flight effects, can be related to bursts of high-frequency electrostatic or electromagnetic fluctuations. We assume that these fluctuations can contribute to the modulation of electron energy spectra either via the scattering of some electron population into the loss cone or via additional energization of resonant electrons in a finite energy range.

\section{Properties and spectra of proton injections observed during the dipolarization}

During the dipolarization event, three short $(\sim$ a few minutes) injections of suprathermal protons $(>50 \mathrm{keV})$ were ob-
Table 3. Time moments of proton injections observed by $\mathrm{C} 4$ during dipolarization.

\begin{tabular}{lll}
\hline \multicolumn{2}{l}{ Proton Injections at $\mathrm{C} 4$} \\
\hline $\begin{array}{l}\text { Time moments } \\
\text { for } 75.3 \mathrm{keV}\end{array}$ & $\begin{array}{l}\text { Time moments } \\
\text { for } 92.2 \mathrm{keV}\end{array}$ & $\begin{array}{l}\text { Time moments } \\
\text { for } 159.7 \mathrm{keV}\end{array}$ \\
\hline$I_{\mathrm{p} 1}: 01: 40: 09.324$ & $01: 40: 05.201$ & $01: 39: 52.834$ \\
$I_{\mathrm{p} 2}: 01: 42: 45.978$ & $01: 42: 45.978$ & $01: 42: 45.978$ \\
$I_{\mathrm{p} 3}: 01: 44: 53.774$ & $01: 44: 53.774$ & $01: 44: 57.897$ \\
\hline
\end{tabular}

served. We identified proton injections as increases in proton flux in, at least, two energy channels by more than 5 times in comparison with the value observed before the flux enhancement.

During the interval of interest a continuous set of data without gaps is provided only by the RAPID instrument on board C4. Figure 5a shows the time profiles of $B_{X}$ and $B_{Z}$ components observed by $\mathrm{C} 4$ for the reference. Figure $5 \mathrm{~b}$ presents the time profile of $B_{Z}^{*}(t)$ calculated as explained in Sect. 3. Figure 5c-f show the dawn-dusk electric field, proton fluxes in a few energy channels $(27.7,75.3,92.2$ and $159.7 \mathrm{kev}$ ), fluxes of suprathermal electrons for reference and the time profile of the power of magnetic field fluctuations at proton gyrofrequency obtained from the wavelet analysis applied to the high-resolution magnetic field data. During the dipolarization event the proton gyrofrequency ranged from 0.051 to $0.56 \mathrm{~Hz}$. The interval of dispersed proton injection is shaded light green and the intervals of dispersionless injections are shaded in grey.

The behavior of suprathermal proton fluxes is different from the behavior of suprathermal electrons. While the suprathermal electrons demonstrate the gradual flux growth along with the growth of the PS $B_{Z}$ field in the course of dipolarization (between 01:38 and 01:55 UT), the level of proton fluxes almost does not change at this timescale. The absence of gradual growth in the energetic protons can be because of Cluster location dawnward of the main magnetic field perturbation, i.e., dawnward of the region of strongest earthward magnetic field gradient. As a result, Cluster observes the growth of electron flux at a large timescale due to the dawnward electron drift, while protons, in the course of their duskward drift, are not recorded by the Cluster spacecraft.

Another difference in the behavior of suprathermal protons and electrons is a smaller number of proton injections observed by $\mathrm{C} 4$ in comparison with a number of electron injections. Only three short proton injections were detected at the $\mathrm{C} 4$ location. The proton injections were only observed during the interval of dipolarization growth. The time moments of the peak flux value observed within each proton injection (marked by dashed vertical lines in Fig. 5) do not coincide with the times of peak flux observed in the electron injections. 

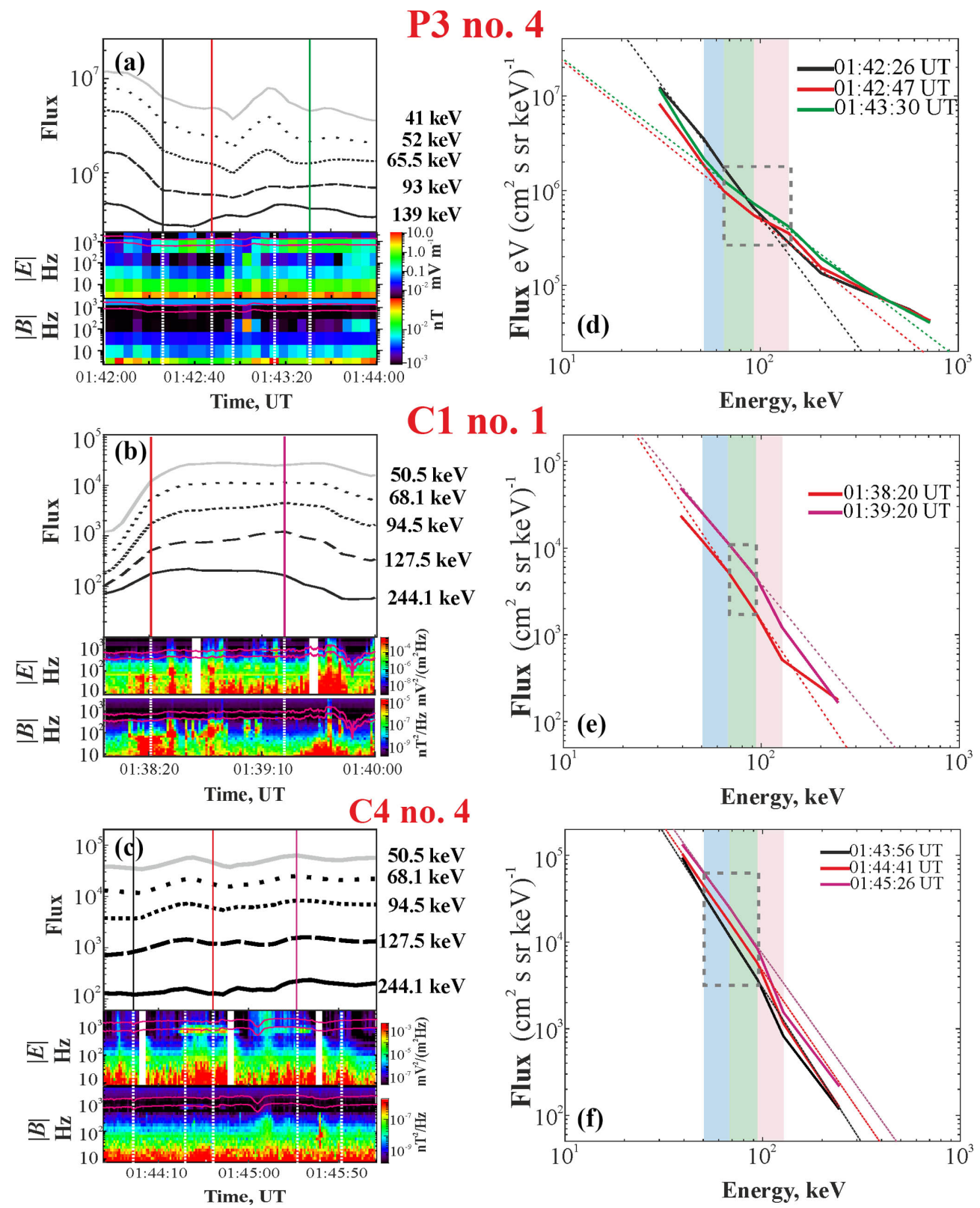

Figure 4. The observations of electron fluxes, spectra and wave activity obtained by P3, C1 and C4 spacecraft. Panels (a-c) show the time profiles of electron fluxes and frequency spectrograms of the power density of electric and magnetic field fluctuations observed by a given spacecraft within short time intervals around the moments marked by red vertical lines in Fig. 3 . The solid magenta lines in frequency spectrograms display the time profiles of $f_{\text {ce }}$ and $2 f_{\text {ce. }}$. Panels $(\mathbf{d}-\mathbf{f})$ show the electron spectra observed by a given spacecraft at the time moments indicated in the left plots by vertical lines of the corresponding color. The colored bars shows three energy ranges for which the spectral index $\gamma$ was calculated. The dashed bars indicate the transient features in energy spectra.

All three proton injections except $I_{\mathrm{p}} 3$ were detected at the trailing edges of the corresponding $B_{Z}$ pulses and the associated $E_{Y}$ bursts (see Fig. $5 \mathrm{a}-\mathrm{d}$ and the exact time moments of proton injections listed in Table 3). Namely, the proton injection $I_{\mathrm{p}} 1$ was observed at 01:40:09 UT just after the $B_{Z}$ pulse marked as DF1 in Table 2. This injection was dispersed as was the electron injection $I_{\mathrm{e}} 2$ (see Table 2) observed before this pulse. We can suggest that both electrons and pro- tons were accelerated by the strong $E_{Y}$ field associated with the $B_{Z}$ pulse. Electrons, being adiabatic, drifted towards the leading edge of the pulse due to the gradient drift, and thus, they were observed before the pulse. Conversely, protons experienced nonadiabatic acceleration by the $E_{Y}$ field and propagated towards the duskward (i.e., trailing) edge of the pulse, and, thus, were observed after the pulse. 


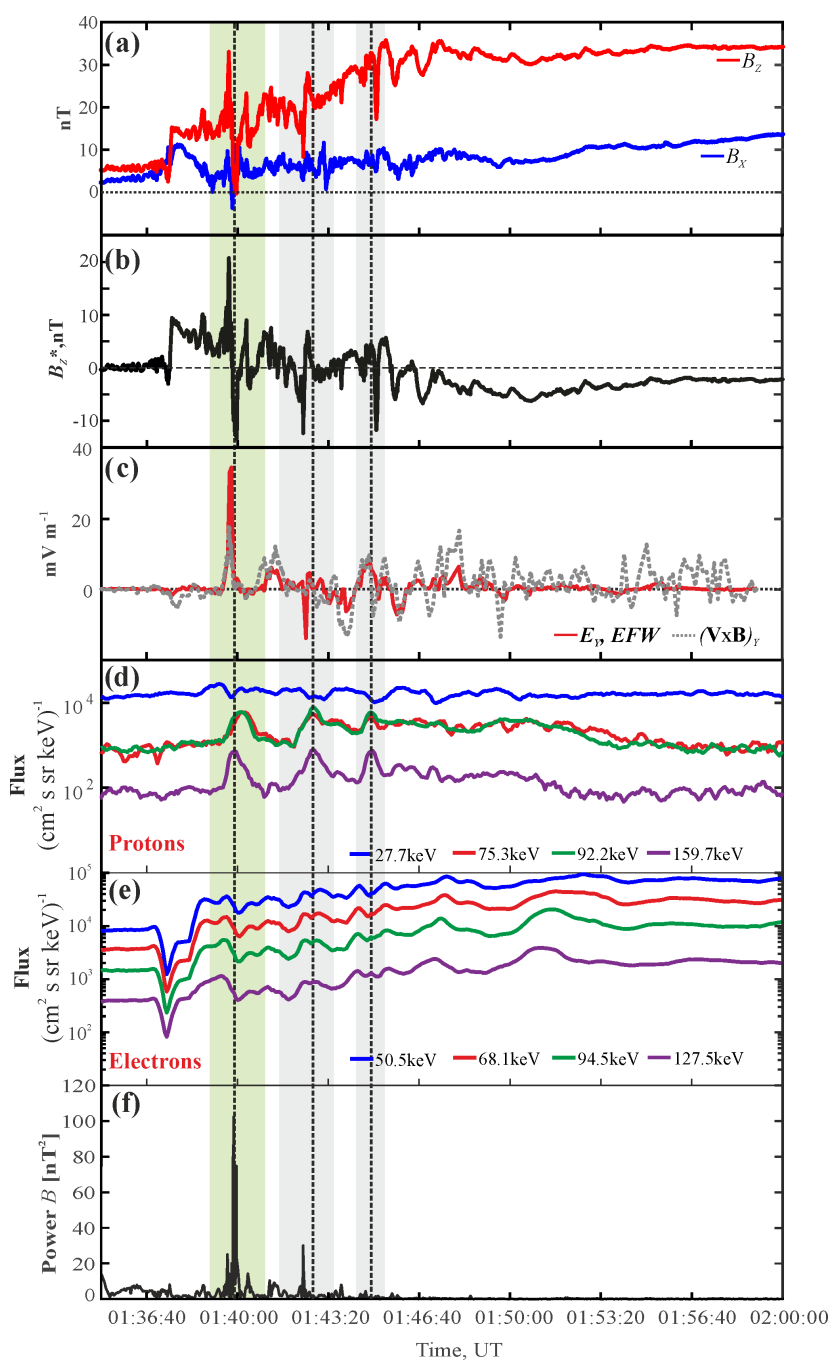

Figure 5. From top to bottom the following data observed by $\mathrm{C} 4$ spacecraft are shown: the time profiles of $B_{X}$ and $B_{Z}$ components (a); the time profile of $B_{Z} *$ (b; see explanations in the text), the dawn-dusk electric field (c), proton fluxes in several energy channels (d), fluxes of suprathermal electrons for the reference (e) and the time profile of the power of magnetic field fluctuations at proton gyrofrequency (f). The interval of the dispersed proton injection is shaded light yellow and the intervals of dispersionless injections are shaded grey. The moments of peak proton flux in each injection are shown by vertical dashed lines.

The two following proton injections $I_{\mathrm{p}} 2$ and $I_{\mathrm{p}} 3$ are dispersionless (see Fig. 5c) and observed at 01:42:46 and 01:44:54 UT, respectively (see Table 3 ). The $I_{\mathrm{p}} 2$ injection was observed behind the $B_{Z}$ pulse (DF3 in Table 2) and the $I_{\mathrm{p}} 3$ injection was detected at the next $B_{Z}$ pulse (DF4 in Table 2). The intermittency in observations of electron and proton injections can be caused by the complicated structure of the flux pile up region consisting of multiple pulses moving one after another $B_{Z}$. The presence of multiple localized $B_{Z}$ pulses results in a complicated multiscale pattern of magnetic gradients, which affect electron drift paths and cause the formation of multiple and short electron injections rather than a monotonic increase in suprathermal electron flux.

Contrary to electrons, proton injections were only associated with the strongest pulses of the $B_{Z}$ and $E_{Y}$ fields observed during the dipolarization growth (see Fig. 5a-c). It is also worth noting that proton injections, except the first one, do not coincide with the peaks of the power of magnetic field fluctuations at proton gyrofrequency. This means that in this event the resonant interactions of protons with low-frequency electromagnetic waves do not contribute much to the proton acceleration.

Another feature of proton injections is the decreases in flux of $27.7 \mathrm{keV}$ protons observed simultaneously with the peaks in more energetic proton fluxes. Figure 6 shows proton fluxes (left plots) and energy spectra (right plots) observed within each injection marked in Fig. $5\left(I_{\mathrm{p}} 1-I_{\mathrm{p}} 3\right)$. In the first injection $\left(I_{\mathrm{p}} 1\right)$ a bump at energy $W \sim 90 \mathrm{keV}$ started to be observed in the proton spectrum at 01:38:22 UT (the yellow spectrum in the right of $I_{\mathrm{p}} 1$ plot). Later, as the flux of suprathermal protons increases, the bump still remains in the spectrum until the end of injection.

A similar feature was observed in the dispersionless injections $I_{\mathrm{p}} 2$ and $I_{\mathrm{p}} 3$. At the time moment corresponding to the peak flux the most pronounced bump was observed in the proton spectra at $W \sim 90 \mathrm{keV}$ (see red spectra in the right of plots $I_{\mathrm{p}} 2$ and $I_{\mathrm{p}} 3$ in Fig. 6). The observed transformation of proton spectra during the injections indicates the selective nonadiabatic acceleration of some part of the lower energy proton population up to $\sim 90 \mathrm{keV}$, which results in some decrease in the lower energy proton flux and in the formation of a bump near $90 \mathrm{keV}$.

\section{Discussion}

\subsection{Summary of the dipolarization event features}

In the present study we investigate the dynamics and spectra of suprathermal $(>50 \mathrm{keV}$ ) electron and proton fluxes simultaneously observed by the THEMIS P3 probe and the Cluster spacecraft during dipolarization in the near-Earth tail (at $X$ $\sim-7--9 R_{\mathrm{E}}$ ) on 20 July 2013 between 01:35 and 01:55 UT. In this event the satellites were mainly separated in the $Y$ direction, which allowed for the multipoint analysis of the particle fluxes and magnetic fields in the azimuthal direction. Before discussing the behavior of energetic electron and proton fluxes and their spectra during the dipolarization we summarize the observed features of this event.

1. The dipolarization was caused by braking and diversion of multiple BBFs that arrived in the near-Earth tail.

2. The onset of dipolarization propagated dawnward at $V_{\text {prop }} \sim 130 \mathrm{~km} \mathrm{~s}^{-1}$. 

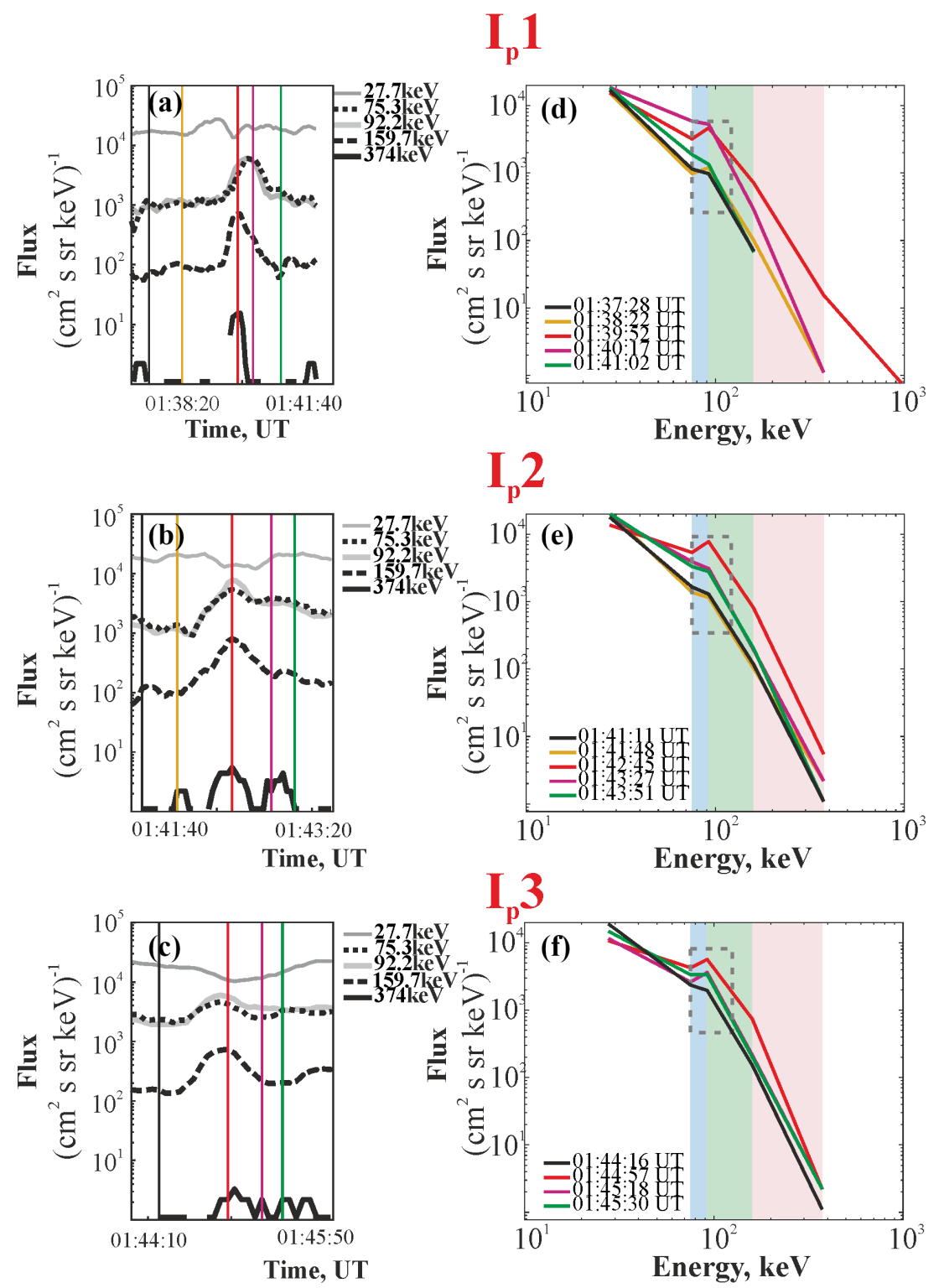

Figure 6. The observations of proton fluxes and spectra by the $\mathrm{C} 4$ spacecraft. The format is similar to the format of Fig. 4 except for wave data.

3. The magnetic field perturbation during the dipolarization event included a gradual growth of the positive $B_{Z}$ field, which was observed by all spacecraft at a similar timescale $(\sim 13 \mathrm{~min})$, and several strong pulses of the $B_{Z}$ with amplitude $\geq 5 \mathrm{nT}$ detected at smaller timescales ( $\leq 1 \mathrm{~min}$ ) during the gradual $B_{Z}$ growth.

4. The strong $B_{Z}$ pulses had characteristics typical of DFs, such as the rapid increase of the magnetic field $B_{Z}$ component preceded by small negative $B_{Z}$ variation, the decrease in density behind the front and the decrease in plasma pressure while magnetic pressure increases across the front (e.g., Shiokawa et al., 2005; Zhou et al., 2009; Runov et al., 2009) (see Fig. 2).
5. The negative variation of the horizontal component of the ground magnetic field $\left(B_{\mathrm{N}}\right)$ started to be observed near the THEMIS P3 footpoint simultaneously with the dipolarization onset at the P3 location. The negative variation of the $B_{\mathrm{N}}$ occurred at a similar timescale to the dipolarization growth in the near-Earth PS. Similarly to the PS dipolarization, the perturbation of the ground magnetic field propagated dawnward.

A dipolarization in the near-Earth tail can be triggered by the development of plasma instability(ies) (e.g., Lui, 1996; Lui et al., 2008) and/or by the BBF braking (e.g., Sergeev et al., 2012 and references therein). In the event analyzed in the present paper the onset of dipolarization observed by the P3 


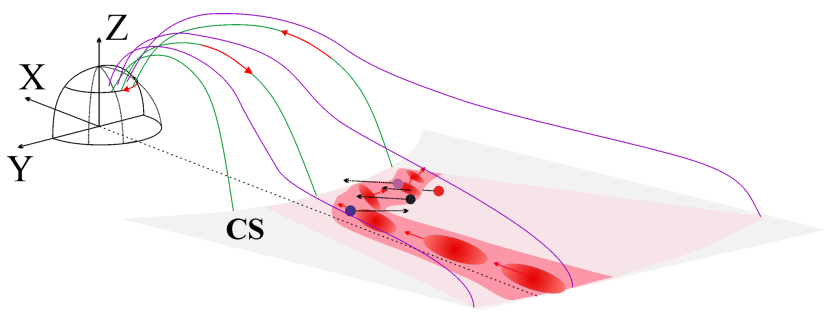

Figure 7. A diagram of the observed magnetic structure of the flux pile up region in the near-Earth PS. An area shaded light pink represents the perturbed CS. The localized area shaded dark pink displays a channel in which the multiple BBFs with the embedded DFs (shown by red ovals) propagate. The direction of DFs' motion is shown by red arrows. P3 probe and Cluster spacecraft are shown by colored dots and their trajectories during the event are shown by black dotted arrows. Near the P3 and Cluster locations the multiple BBFs are braked and diverted. The system of field-aligned currents and westward electrojet is shown by red arrows.

probe and the Cluster spacecraft was associated with the arrival and braking/deflection of multiple BBFs. Multiple $B_{Z}$ pulses observed after the onset had the characteristics typical of DFs. All these features are unique to the so-called secondary dipolarization, the term suggested by Nakamura et al. (2009) for dipolarizations that evolve in the near-Earth PS due to the magnetic flux pile up caused by the arrival of multiple BBFs with the embedded DFs (see diagram shown in Fig. 7). The dipolarization was followed by the development of large-scale (both in space and time) perturbation of the cross-tail electric current and the corresponding formation of the SCW discussed before by e.g., Lui (2011) and Sergeev et al. (2012).

\subsection{The dynamics and acceleration of suprathermal electrons during the dipolarization}

During the dipolarization the fluxes of suprathermal electrons and protons exhibited different dynamics, suggesting different mechanisms responsible for their energization. The beginning of the increase in energetic electron flux, i.e., the electron injection boundary, was associated with the onset of dipolarization and it propagated dawnward along with the dipolarization. After the onset the time profiles of the energetic electron fluxes $J_{\mathrm{e}}$ were very similar to the time profiles of both the $B_{Z}(t)$ and the $|B|(t)$ in the near-Earth PS. Indeed, the correlation coefficients (CCs) calculated between $J_{\mathrm{e}}(t)$ at all energy ranges presented in Fig. 3 and the corresponding $|B|(t)$ observed during the dipolarization (at 01:37:30-01:50 UT) are $\geq 0.8$ both at P3 and at Cluster locations. The time profiles of $J_{\mathrm{e}}$ exhibited multiple enhancements (or injections) at short timescales, which were associated with multiple $B_{Z}$ pulses observed during the dipolarization growth. The majority of electron injections associated with the $B_{Z}$ pulses were dispersionless, which indicates the local electron acceleration at the $B_{Z}$ pulses.

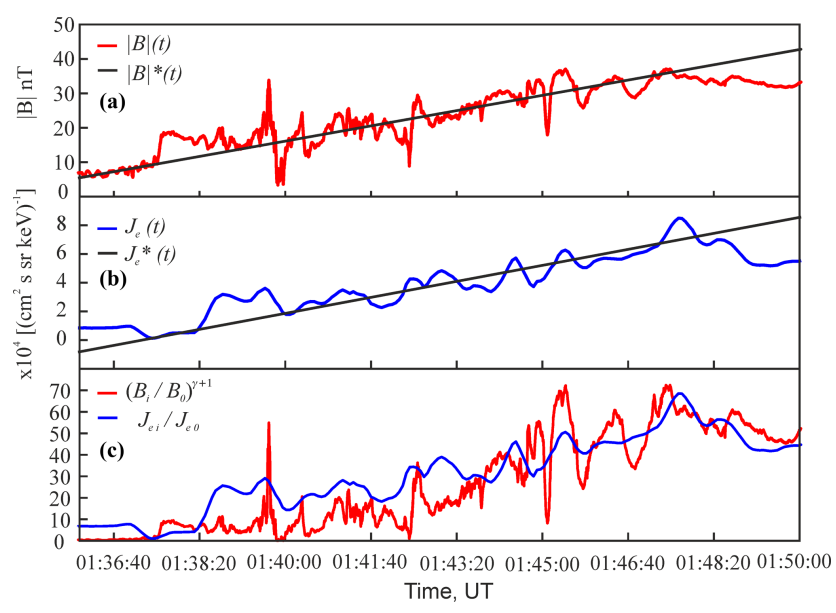

Figure 8. Panels $(\mathbf{a}-\mathbf{c})$ show the time profiles of the absolute value of the magnetic field $|B|(t)$ and electron flux $J_{\mathrm{e}}(t)$ at $50.5 \mathrm{keV}$ displayed along with the linear approximations $|B|^{*}(t)$ and $J_{\mathrm{e}}^{*}(t)$, respectively (for explanation, see Sect. 6). In (c) the time profiles of $J_{\mathrm{ei}}(t) / J_{\mathrm{e} 1}$ and $\left(B_{i}(t) / B_{1}\right)^{\gamma+1}$ are shown (for explanation, see Sect. 6.2).

The resemblance between the dynamics of suprathermal electron fluxes and the $B_{Z}(t)$ indicates the significant contribution of the betatron mechanism in the electron energization discussed before by e.g., Asano et al. (2010), Fu et al. (2011), Birn et al. (2013, 2014) and Gabrielse et al. (2016). To check this assumption we performed a test on the betatron energization of electrons during the dipolarization.

The gradual increase in energetic electron flux $\left(J_{\mathrm{e}}\right)$ and in $|B|$ can be described by linear functions: $|B|(t)=0.045 t+$ 9.5 and $J_{\mathrm{e}}(t)=112 t+1200$. We show these dependencies at two top panels in Fig. 8 along with the observed time profiles. According to the adiabatic theory discussed by e.g., Pan et al. (2012), the relation between the electron flux $\left(J_{\mathrm{e} 2}\right)$ averaged for $1 \mathrm{~min}$ after the end of dipolarization (at 01:48-01:49 UT) and the electron flux $\left(J_{e 1}\right)$ averaged for $1 \mathrm{~min}$ before dipolarization onset (at 01:36:30-01:37:30 UT) is $J_{\mathrm{e} 2} / J_{\mathrm{e} 1}=\left(B_{2} / B_{1}\right)^{\gamma+1}$, where $B_{2}$ and $B_{1}$ are absolute values of the magnetic field averaged for the same intervals as $J_{\mathrm{e} 2}$ and $J_{\mathrm{e} 1}$. We calculate that $J_{e 2} / J_{\mathrm{e} 1} \sim 55$ and $\left(B_{2} / B_{1}\right)^{\gamma+1}$ $\sim 58$, for $\gamma=2.7$ (we used $\gamma$ calculated for the energy range of $50.5-68.1 \mathrm{keV}$ and averaged for the interval of the dipolarization growth). The similarity of both values means that the gradual growth of the electron flux observed at a large timescale is mainly caused by the betatron acceleration.

To check the operation of adiabatic mechanism in the course of dipolarization we plot the time profiles of $J_{\mathrm{ei}}(t) / J_{\mathrm{e} 1}$ and $\left(B_{i}(t) / B_{1}\right)^{\gamma+1}$ in the bottom panel of Fig. 8; here $J_{\mathrm{ei}}(t)$ and $B_{\mathrm{i}}(t)$ are the electron flux in the energy range of 50.5-68.1 keV and the value of magnetic field observed at each time moment during the dipolarization, respectively. It is seen that the increase in the electron flux observed at a large timescale, which includes the entire dipo- 
larization growth, can be roughly explained by the betatron mechanism. However, during the gradual magnetic field growth there are significant deviations of $J_{\mathrm{ei}}(t) / J_{\mathrm{e} 1}$ from $\left[\left(B_{i}(t) / B_{1}\right)\right]^{\gamma+1}$. These deviations can be due to gradient drifts and/or scattering of electrons into the loss cone.

Gabrielse et al. (2016) showed the importance of gradient and curvature drifts in the modulation of the energetic electron flux. In our dipolarization event the presence of multiple localized $B_{Z}$ pulses propagating both in the radial and azimuthal (dawnward) directions leads to the formation of complicated spatial distribution of the magnetic gradients in the near-Earth PS. Thus, the observed complicated dynamics of the energetic electron fluxes can be a result of combination of the local betatron acceleration at the $B_{Z}$ pulses and the electron drifts in the multiscale magnetic structure formed in the near-Earth PS due to magnetic flux pile up.

The spectral index $\gamma$ calculated for three energy ranges of suprathermal electrons occasionally experienced pronounced variations during the dipolarization. Some of these variations were observed during the dispersed electron injections, and, thus, they can be related to the time-of-flight effect caused by energy-dependent electron drifts. Generally the betatron acceleration does not affect the slope of the energy spectrum (e.g., Pan et al., 2012). However, the variations of $\gamma$ observed during the dispersionless injections cannot be explained by the time-of-flight effect and should be caused by the changes in the electron energy distribution in a finite energy range. The other nonadiabatic mechanisms can be responsible for these features. Birn et al. (2012) suggested that an additional energization of some part of the electron population can be due to electron interactions with waves generated in the course of dipolarization. Panov et al. (2013) reported that the resonant interactions of electrons with highfrequency electromagnetic fluctuations can provide an additional scattering of some part of the electron population into the loss cone. Both electron energization and losses affect the $\gamma$. In the dipolarization event analyzed in the present study those $\gamma$ variations which cannot be explained by the time-of-flight effect were observed along with the bursts in high-frequency wave activity representing either the broadband electromagnetic emission in frequency range from a few $\mathrm{Hz}$ and up to $f_{\text {ce }}$ (see the intervals $\mathrm{C} 1$ no. 1 in Fig. 4 ) or the broadband electrostatic fluctuations including ECH (see the interval P3 no. 4 and $\mathrm{C} 4$ no. 4 in Fig. 4). Thus, we may assume that besides the time-of-flight effects caused by e.g., energy-dependent gradient drifts of electrons in the flux pile up region, the variations of electron energy spectra can be caused by the interactions of resonant electrons with highfrequency fluctuations, leading to the additional electron energization and/or to the scattering of some part of the electron population into the loss cone. This assumption requires a quantitative verification, which we are going to perform in future studies.

\subsection{The dynamics and acceleration of suprathermal protons during the dipolarization}

The dynamics of suprathermal protons during the dipolarization was drastically different from the dynamics of energetic electrons. In contrast with electrons the fluxes of suprathermal protons $\left(J_{\mathrm{p}}\right)$ did not experience the gradual growth during dipolarization. The correlation coefficients (CCs) calculated between $J_{\mathrm{p}}(t)$ at all energy ranges presented in Fig. 5 and the corresponding $|B|(t)$ observed during the dipolarization (at 01:37:30-01:50 UT) were $<0.3$.

Only three proton injections were identified during the dipolarization. The first one was not related to the onset of dipolarization but it was observed after the onset simultaneously with the first strong $B_{Z}$ pulse and the associated burst of the dawn-dusk electric field $\left(E_{Y}\right)$ (see Fig. 5). The next two injections of energetic protons were also only associated with the strong $B_{Z}$ pulses. As a result a poor correlation between the proton flux $J_{\mathrm{p}}$ at all analyzed energy ranges and the $|B|(t)(C C<0.3)$ was observed at the $\mathrm{C} 4$ location.

Using the timing analysis we estimate the propagation velocity and the spatial scale of the $B_{Z}$ pulses ( $\left.L_{\text {pulse }}\right)$ as well as the thicknesses of their fronts, $L_{F}$, and compare these values with the gyroradius of thermal protons $\left(\rho_{\mathrm{T}}\right)$ observed at the moment of the corresponding $B_{Z}$ pulse (see Table 4$)$. We revealed a decrease in propagation velocity of the $B_{Z}$ pulses as the dipolarization progresses in the PS. The spatial scale of $B_{Z}$ pulses, $L_{\text {pulse }}$, is typically of the order of a few $\rho_{\mathrm{T}}$, while the $L_{\mathrm{F}}$ is less than $\rho_{\mathrm{T}}$ for all analyzed pulses. This is in agreement with previous results reported for the DFs (e.g., Angelopoulos et al., 2013; Balikhin et al., 2014).

Since the $B_{Z}$ pulses observed during the dipolarization growth represent the magnetic structures formed at subproton kinetic scales, it is natural to expect the nonadiabatic proton acceleration in these structures. This mechanism was studied before for an isolated earthward-propagating DF by using the test particle simulations (e.g., Artemyev et al., 2012; Ukhorskiy et al., 2013). Artemyev et al. (2012), using the observations of energetic ion spectra by the DOK instrument (Lutsenko et al., 1998) on board the Interball-1 spacecraft, simultaneously showed the presence of a bulge in ion spectra at $\sim 100 \mathrm{keV}$ with the earthward-propagating isolated DF. The authors suggested that this bulge was formed due to the resonant nonadiabatic acceleration of ions by the dawn-dusk electric field associated with the DF. Ukhorskiy et al. (2013) considered a more complicated acceleration scenario including a full or partial trapping of nonadiabatic ions in the region of minimum $B_{Z}$ field preceding a moving DF. Authors showed that due to the trapping some population of thermal ions can gain energy up to $100 \mathrm{keV}$ if the electric field associated with the $\mathrm{DF}$ is about several $\mathrm{mV} \mathrm{m}^{-1}$.

In three proton injections observed in our event a bulge was detected in the energy range 75.3-92.2 keV. Within each injection the bulge was observed at the moment of peak in energetic proton flux and simultaneously with some decrease 
Table 4. Propagation velocity ( $V_{\text {prop}}$ ) of the $B_{Z}$ pulses (DFs), the spatial scale of the pulse structure $\left(L_{S}\right)$ and the thickness of the fronts $\left(L_{\mathrm{F}}\right)$ calculated for several $B_{Z}$ pulses observed by $\mathrm{C} 1$ and $\mathrm{C} 4$ during the dipolarization.

\begin{tabular}{lrrr}
\hline DF nos. (see Table 2) & $V_{\text {prop }} \mathrm{km} \mathrm{s}^{-1}$ & $L_{\text {pulse }}, \mathrm{km}$ & $L_{\mathrm{F}}, \mathrm{km}$ \\
\hline DF2 by C1 DF1 by C4 & 222 & $2748 \sim 6.0 \rho_{\mathrm{T}}$ & $366<\rho_{\mathrm{T}}\left(\rho_{\mathrm{T}} \sim 465 \mathrm{~km}\right)$ \\
DF4 by C1 DF3 by C4 & 110 & $713 \sim 1.5 \rho_{\mathrm{T}}$ & $408<\rho_{\mathrm{T}}\left(\rho_{\mathrm{T}} \sim 460 \mathrm{~km}\right)$ \\
DF5 by C1 DF4 by C4 & 131 & $667 \sim 2.0 \rho_{\mathrm{T}}$ & $340<\rho_{\mathrm{T}}\left(\rho_{\mathrm{T}} \sim 390 \mathrm{~km}\right)$ \\
DF6 by C1 DF5 by C4 & 52 & $1065 \sim 3.0 \rho_{\mathrm{T}}$ & $311<\rho_{\mathrm{T}}\left(\rho_{\mathrm{T}} \sim 360 \mathrm{~km}\right)$ \\
\hline
\end{tabular}

in flux of lower energy protons ( $27.7 \mathrm{keV}$; see Fig. 6). The decrease in $27.7 \mathrm{keV}$ proton flux was related to the decrease in density of thermal protons behind the DF. However, the simultaneous increase in energetic proton flux in a finite energy range, which leads to the bulge formation, suggests the resonant nonadiabatic acceleration of some part of thermal proton population.

During the first proton injection the burst of strong $E_{Y}$ electric field up to $\sim 35 \mathrm{mV} \mathrm{m}^{-1}$ was observed. This field correlates with the $-(V \times B)_{Y}$ field, although it has a larger amplitude (see Fig. 5). To gain $\sim 70 \mathrm{keV}$ a nonadiabatic proton should pass along the observed $E_{Y}$ field a distance of $\Delta Y \sim 2000 \mathrm{~km}$. This spatial scale is comparable with the spatial scale of the $B_{Z}$ pulse preceding this injection. The puzzling feature of the first injection is the observation of energy dispersion. The energy dispersion can be caused by the time-of-flight effect due to the direct particle propagation from a remote source, or it can be due to the energydependent gradient drift. The latter is not applicable for nonadiabatic protons. Assuming the local proton acceleration by the $E_{Y}$ pulse we may suggest that this dispersion can be due to the spatial effect related to the peculiarities of interaction and trapping of protons with different gyroradii (energies) in the pronounced magnetic dip behind the $B_{Z}$ pulse.

The other proton injections were dispersionless and they were associated with the bursts of $E_{Y}$ field with much smaller amplitudes than the first one $\left(\sim 5 \mathrm{mV} \mathrm{m}^{-1}\right.$ for the second injection and $\sim 10 \mathrm{mV} \mathrm{m}^{-1}$ for the third injection). However, the proton spectra observed in these injections demonstrate features similar to the first one: a bulge in the energy range of $75.3-92.2 \mathrm{keV}$ is clearly observed at the moment of peak flux of energetic protons simultaneously with the decrease in flux of lower energy protons $(27.7 \mathrm{keV})$. In these cases, to gain the energy corresponding to the energy of the bulge, the nonadiabatic protons should pass a distance along the $E_{Y}$ field of $\sim 1.5-2.5 R_{\mathrm{E}}$. These distances are comparable with the previous estimations of the spatial scale of BBFs in the $Y$ direction (e.g., Nakamura et al., 2004). Since these proton injections were observed during the intervals of bulk flow diversion/oscillation, we can assume that the spatial scales of the $E_{Y}$ field bursts were comparable with the spatial scale of the flow channel(s) and, thus, were large enough to accelerate nonadiabatic protons to $\sim 70-90 \mathrm{keV}$.
Finally, we should note that the magnetic structure of dipolarization observed in our event is different from the ones considered in the studies by Artemyev et al. (2012) and Ukhorskiy et al. (2013). However, if one subtracts a largescale $B_{Z}$ variation related to the gradual $B_{Z}$ growth from the $B_{Z}(t)$ profile observed during the dipolarization, then one obtains the time sequence of multiple positive $B_{Z}$ pulses with short duration separated by the negative $B_{Z}$ variations (see Fig. 5b). This pattern resembles the time sequence of multiple DFs. The process of ion nonadiabatic acceleration in the course of interactions with such multiple magnetic structures deserves a separate modeling study.

\section{Conclusions}

In the present paper we analyzed the properties and spectra of suprathermal $(>50 \mathrm{keV})$ electron and proton injections observed during dipolarization in the near-Earth PS at $X \sim-7--9 R_{\mathrm{E}}$ by the THEMIS P3 probe and the Cluster spacecraft. The dipolarization was associated with braking and azimuthal diversion of multiple bursty bulk flows with DFs and it was followed by SCW formation. During the dipolarization the fluxes of suprathermal electrons and protons exhibited contrasting dynamics, suggesting different mechanisms of their energization.

Energetic electron fluxes exhibited a complicated dynamics, which was very similar to the dynamics of the magnetic field during the dipolarization. The quantitative analysis of the observed variations of the magnetic field and electron fluxes demonstrated that at the large timescale, namely, at the timescale of the gradual dipolarization growth, the increase in energetic electron flux can be explained by the betatron acceleration. However, deviations from the betatron scenario were observed at shorter timescales. These deviations can be due to the gradient drifts of electrons in the magnetic flux pile region and/or due to electron scattering into the loss cone.

The spectral index $\gamma$ of suprathermal electrons experienced strong transient variations during the dipolarization growth. Some of these variations were associated with dispersed injections and, thus, can be explained by energydependent drifts of electrons in the magnetic flux pile up region. However, at some moments the observed $\gamma$ variations can be related to the nonadiabatic effects of electron interactions with high-frequency electromagnetic or electro- 
static fluctuations, leading either to the energization of resonant electrons in a finite energy range or to the scattering of some electron population into the loss cone. This assumption requires quantitative verification, which we are going to perform in future studies.

Contrary to the energetic electrons, the dynamics of suprathermal protons was not similar to the dynamics of the magnetic field during the dipolarization. A few proton injections observed during the dipolarization growth were only associated with the strong $B_{Z}$ pulses. During these injections a bulge was clearly observed in the energy spectra of protons in a finite energy range of $\sim 70-90 \mathrm{keV}$. This feature can be explained by the nonadiabatic resonant interactions of some part of the thermal proton population with the fronts of $B_{Z}$ pulses. In the course of these interactions thermal protons can be partially trapped behind the fronts and accelerated up to the energies of the bulge by the observed bursts of the dawn-dusk electric field associated with the $B_{Z}$ pulses. The suggested mechanism resembles a mechanism of protons' acceleration in the course of nonadiabatic interactions with a moving isolated DF.

Data availability. In this paper we only used open-access data. The Cluster data were downloaded from the Cluster Science Archive version 1.2.1 at http://www.cosmos.esa.int/web/csa (last access: 5 March 2018). To obtain the data, one should start the CSA GRAPHICAL USER INTERFACE, and then to download the data, the particular instrument and time interval should be selected. The THEMIS data were downloaded from the Coordinated Data Analysis Web (CDAWeb) https://cdaweb.sci.gsfc.nasa.gov/index.html (last access: 4 March 2018) and from http://themis.ssl.berkeley.edu/ index.shtml (last access: 4 March 2018). Ground magnetometer data were retrieved from the SuperMag website at http://supermag. jhuapl.edu (last access: 13 February 2018).

Competing interests. The authors declare that they have no conflict of interest.

Acknowledgements. We acknowledge NASA contract NAS502099 and Vassilis Angelopoulos for use of data from the THEMIS Mission. Specifically we thank Principal Investigators of FGM, ESA and SST instruments. We also thank Principal Investigators of FGM, CIS, RAPID, PEACE instruments of Cluster mission. We acknowledge principal investigator Rico Behlke who provided data from the Greenland Magnetometer Array (DTU Space) to SuperMag. The work of Andrey Yu. Malykhin and Elena E. Grigorenko was supported by the Russian Science Foundation (grant no. 14-12-00824). The work of Elena A. Kronberg and Ludmila Kozak was supported by the Volkswagen Foundation (grant Az 90 312). Elena A. Kronberg and Patrick W. Daly acknowledge the "Deutsches Zentrum für Luft und Raumfahrt (DLR)" for the support of the RAPID instrument under grant 50 OC 1602. The work of Rositza Koleva was partially supported by the agreement between RAS and BAS on fundamental space research. The re- search of Natalia Y. Ganushkina was partly funded by the European Union's Horizon 2020 Research and Innovation programme under grant agreement no. 637302 PROGRESS. The work of Natalia Y. Ganushkina in Michigan was also partly supported by the National Aeronautics and Space Administration under Grant Agreements No. NNX14AF34G (issued through the ROSES-2013 Program) and No. NNX17AI48G, NNX17AB87G, and 80NSSC17K0015 (issued through ROSES-2016 Program). Natalia Y. Ganushkina thank the Academy of Finland for the support of the Space Cooperation in the Science and Technology Commission between Finland and Russia (TT/AVA). Elena A. Kronberg acknowledges Drew Turner and ISSI for hosting 70 the team on "Understanding energetic particle injections and their effect on Earth's outer radiation belt electrons using multipoint observations".

The topical editor, Christopher Owen, thanks the two anonymous referees for help in evaluating this paper.

\section{References}

Angelopoulos, V.: The THEMIS mission, Space Sci. Rev., 598, 534, https://doi.org/10.1007/s11214-008-9336-1, 2008.

Angelopoulos, V., Baumjohann, W., Kennel, C. F., Coroniti, F. V., Kivelson, M. G., Pellat, R., Walker, R. J., Lühr, H., and Paschmann, G.: Bursty bulk flows in the inner central plasma sheet, J. Geophys. Res., 97, 4027-4039, https://doi.org/10.1029/91JA02701, 1992.

Angelopoulos, V., Runov, A., Zhou, X.-Z., Turner, D. L., Kiehas, S. A., Li, S.-S., and Shinohara, I.: Electromagnetic energy conversion at reconnection front, Science, 341, 1478-1482, https://doi.org/10.1126/science.1236992, 2013.

Apatenkov, S. V., Sergeev, V. A., Kubyshkina, M. V., Nakamura, R., Baumjohann, W., Runov, A., Alexeev, I., Fazakerley, A., Frey, H., Muhlbachler, S., Daly, P. W., Sauvaud, J.-A., Ganushkina, N., Pulkkinen, T., Reeves, G. D., and Khotyaintsev, Y.: Multi-spacecraft observation of plasma dipolarization/injection in the inner magnetosphere, Ann. Geophys., 25, 801-814, https://doi.org/10.5194/angeo-25-801-2007, 2007.

Apatenkov, S. V., Sergeev, V. A., Amm, O., Baumjohann, W., Nakamura, R., Runov, A., Rich, F., Daly, P., Fazakerley, A., Alexeev, I., Sauvaud, J. A., and Chotyaintsev, Yu.: Conjugate observation of sharp dynamical boundary in the inner magnetosphere by Cluster and DMSP spacecraft and ground network, Ann. Geophys., 26, 2771-2780, https://doi.org/10.5194/angeo26-2771-2008, 2008.

Artemyev, A. V., Lutsenko, V. N., and Petrukovich, A. A.: Ion resonance acceleration by dipolarization fronts: analytic theory and spacecraft observation, Ann. Geophys., 30, 317-324, https://doi.org/10.5194/angeo-30-317-2012, 2012.

Asano, Y., Shinohara, I., Retinò, A., Daly, P. W., Kronberg, E. A., Takada, T., Nakamura, R., Khotyaintsev, Y. V., Vaivads, A., Nagai, T., Baumjohann, W., Fazakerley, A. N., Owen, C. J., Miyashita, Y., Lucek, E. A., Rème, H.: Electron acceleration signatures in the magnetotail associated with substorms, J. Geophys. Res., 115, A05215, https://doi.org/10.1029/2009JA014587, 2010.

Ashour-Abdalla, M., El-Alaoui, M., Goldstein, M. L., Zhou, M., Schriver, D., Richard, R., Walker, R., Kivelson, M. G., and Hwang, K.: Observations and simulations of non-local acceler- 
ation of electrons in magnetotail magnetic reconnection events, Nat. Phys., 7, 360-365, https://doi.org/10.1038/nphys1903, 2011.

Balikhin, M. A., Runov, A., Walker, S. N., Gedalin, M., Dandouras, I., Hobara, Y., and Fazakerley, A.: On the fine structure of dipolarization fronts, J. Geophys. Res., 119, 6367-6385, https://doi.org/10.1002/2014JA019908, 2014.

Balogh, A., Carr, C. M., Acuña, M. H., Dunlop, M. W., Beek, T. J., Brown, P., Fornacon, K.-H., Georgescu, E., Glassmeier, K.H., Harris, J., Musmann, G., Oddy, T., and Schwingenschuh, K.: The Cluster Magnetic Field Investigation: overview of in-flight performance and initial results, Ann. Geophys., 19, 1207-1217, https://doi.org/10.5194/angeo-19-1207-2001, 2001.

Birn, J.: Magnetotail equilibrium theory - the general 3Dimensional solution, J. Geophys. Res., 92, 11101-11108, 1987.

Birn, J. and Hesse, M.: The substorm current wedge in MHD simulations, J. Geophys. Res., 118, 3364-3376, https://doi.org/10.1002/jgra.50187, 2013.

Birn, J., Thomsen, M. F., Borovsky, J. E., Reeves, G. D., McComas, D. J., Belian, R. D., and Hesse, M.: Substorm ion injections: Geosynhronous observations and test particle orbits in three-dimensional dynamics MHD fields, J. Geophys. Res., 102, 2325-2341, 1997.

Birn, J., Nakamura, R., Panov, E. V., and Hesse, M.: Bursty bulk flows and dipolarization in MHD simulations of magnetotail reconnection, J. Geophys. Res., 116, A01210, https://doi.org/10.1029/2010JA016083, 2011.

Birn, J., Artemyev, A. V., Baker, D. N., Echim, M., Hoshino, M., and Zelenyi, L. M.: Particle acceleration in the magnetotail and aurora, Space Sci. Rev., 173, 49-102, https://doi.org/10.1007/s11214-012-9874-4, 2012.

Birn, J., Hesse, M., Nakamura, R., and Zaharia, S.: Particle acceleration in dipolarization events, J. Geophys. Res., 118, 1960-1971, https://doi.org/10.1002/jgra.50132, 2013.

Birn, J., Runov, A., and Hesse, M.: Energetic electrons in dipolarization events: Spatial properties and anisotropy, J. Geophys. Res., 119, 3604-3616, https://doi.org/10.1002/2013JA019738, 2014.

Cluster Science Archive: Access to the Archive, available at: https: //www.cosmos.esa.int/web/csa, last acces: 5 March, 2018.

Coordinated Data Analysis Web (CDAWeb): Public data from current and past space physics missions, available at: https://cdaweb. sci.gsfc.nasa.gov/index.html/, last acces: 4 March, 2018.

Deng, X., Ashour-Abdalla, M., Zhou, M., Walker, R., El-Alaoui, M., Angelopoulos, V., Ergun, R. E., and Schriver, D.: Wave and particle characteristics of earthward injections associated with dipolarization fronts, J. Geophys. Res., 115, A09225, https://doi.org/10.1029/2009JA015107, 2010.

Ergun, R. E., Goodrich, K. A., Stawarz, J. E., Andersson, L., and Angelopoulos, V.: Large-amplitude electric fields associated with bursty bulk flow braking in the Earth's plasma sheet, J. Gophys. Res., 120, 1832-1844, https://doi.org/10.1002/2014JA020165, 2015.

Forsyth, C., Fazakerley, A. N., Rae, I. J., Watt, C. E. J., Murphy, K., Wild, J. A., Karlsson, T., Mutel, R., Owen, C. J., Ergun, R., Masson, A., Berthomier, M., Donovan, E., Frey, H. U., Matzka, J., Stolle, C., and Zhang, Y.: In situ spatiotemporal measurements of the detailed azimuthal substructure of the substorm current wedge, J. Geophys. Res.-Space Phys., 119, 927-946, https://doi.org/10.1002/2013JA019302, 2014.

Fu, H. S., Khotyaintsev, Y. V., André, M., and Vaivads, A.: Fermi and betatron acceleration of suprathermal electrons behind dipolarization fronts, Geophys. Res. Lett., 38, L16104, https://doi.org/10.1029/2011GL048528, 2011.

Fu, H. S., Khotyaintsev, Y. V., Vaivads, A., André, M., Sergeev, V. A., Huang, S. Y., Kronberg, E. A., and Daly, P. W.: Pitch angle distribution of suprathermal electrons behind dipolarization fronts: A statistical overview, J. Geophys. Res., 117, A12221, https://doi.org/10.1029/2012JA018141, 2012a.

Fu, H. S., Khotyaintsev, Y. V., Vivads, A., André, M., and Huang, S. Y.: Electric structure of dipolarization front at sub-proton scale, Geophys. Res. Lett., 39, L06105, https://doi.org/10.1029/2012GL051274, 2012b.

Fu, H. S., Cao, J. B., Cully, C. M., Khotyaintsev, Y. V., Vaivads, A., Angelopoulos, V., Zong, Q.-G., Santolík, O., Macúšová, E., André, M., Liu, W. L., Lu, H. Y., Zhou, M., Huang, S. Y., and Zhima, Z.: Whistler-mode waves inside flux pileup region: Structured or unstructured?, J. Geophys. Res.-Space Phys., 119, 9089-9100, https://doi.org/10.1002/2014JA020204, 2014.

Gabrielse, C., Angelopoulos, V., Runov, A., and Turner, D. L.: Statistical characteristics of particles injections throughout the equatorial magnetotail, J. Geophys. Res., 119, 2512-2535, https://doi.org/10.1002/2013JA019638, 2014.

Gabrielse, C., Harris, C., Angelopoulos, V., Artemyev, A., and Runov, A.: The role of localized inductive electric fields in electron injections around dipolarizing flux bundles, J. Geophys. Res., 121, 9560-9585, https://doi.org/10.1002/2016JA023061, 2016.

Ganushkina, N. Yu., Pulkkinen, T. I., Bashkirov, V. F., Baker, D. N., and Li, X.: Formation of intense nose structures, Geophys. Res. Lett., 28, 491-494, 2001.

Ganushkina, N. Yu., Pulkkinen, T. I., and Fritz, T.: Role of substorm-associated impulsive electric fields in the ring current development during storms, Ann. Geophys., 23, 579-591, https://doi.org/10.5194/angeo-23-579-2005, 2005.

Ganushkina, N. Yu., Amariutei, O. A., Shpritz, Y. Y., and Liemohn, M. W.: Transport of the plasma sheet electrons to the geostationary distances, J. Geophys. Res., 118, 82-98, https://doi.org/10.1029/2012JA017923, 2013.

Ge, Y. S., Raeder, J., Angelopoulos, V., Gilson, M. L., and Runov, A.: Interaction of dipolarization fronts within multiple bulk flows in global MHD simulations of a substorm on 27 February 2009, J. Geophys. Res., 116, A00123, https://doi.org/10.1029/2010JA015758, 2011.

Grigorenko, E. E., Kronberg, E. A., Daly, P. W., Ganushkina, N. Yu., Lavraud, B., Sauvaud, J.-A., and Zelenyi, L. M.: Origin of low proton-to-electron temperature ratio in the Earth's plasma sheet, J. Geophys. Res.-Space Phys., 121, 9985-10004, https://doi.org/10.1002/2016JA022874, 2016.

Grigorenko, E. E., Kronberg, E. A., and Daly, P. W.: Heating and Acceleration of Charged Particles during Magnetic Dipolarizations, Cosmic Res., 55, 57-66, 2017.

Grigorenko, E. E., Dubyagin, S., Malykhin, A. Yu., Khotyaintsev, Yu, V., Kronberg, E. A., Lavraud, B., and Ganushkina, N. Yu.: Intense current structures observed at electron kinetic scales in the near-Earth magnetotail during dipolarization and substorm 
current wedge formation, Geophys. Res. Lett., 45, 602-611, https://doi.org/10.1002/2017GL076303, 2018.

Hwang, K.-J., Goldstein, M. L., Lee, E., and Pickett, J. S.: Cluster observations of multiple dipolarization fronts, J. Geophys. Res., 116, A00I32, https://doi.org/10.1029/2010JA015742, 2011.

Imada, S., Nakamura, R., Daly, P. W., Hoshino, M., Baumjohann, W., Muhlbachler, S., Balogh, A., and Reme, H.: Energetic electron acceleration in the downstream reconnection outflow region, J. Geophys. Res., 112, A03202, https://doi.org/10.1029/2006JA011847, 2007.

Khotyaintsev, Y. V., Cully, C. M., Vaivads, A., André, M., and Owen, C. J.: Plasma jet braking: Energy dissipation and nonadiabatic electrons, Phys. Rev. Lett., 106, 165001, https://doi.org/10.1103/PhysRevLett.106.165001, 2011.

Khotyaintsev, Yu. V., Divin, A., Vaivads, A., André, M., and Markidis, S.: Energy conversion at dipolarization fronts, Geophys. Res. Lett., 44, 1234-1242, https://doi.org/10.1002/2016GL071909, 2017.

Kronberg, E. A. and Daly, P. W.: Spectral analysis for wide energy channels, Geosci. Instrum. Method. Data Syst., 2, 257-261, https://doi.org/10.5194/gi-2-257-2013, 2013.

Kronberg, E. A., Ashour-Abdalla, M., Dandouras, I., Delcourt, D. C., Grigorenko, E. E., Kistler, L. M., Kuzichev, I. V., Liao, J., Maggiolo, R., Malova, H. V., Orlova, K. G., Peroomian, V., Shklyar, D. R., Shprits, Y. Y., Welling, D. T., and Zelenyi, L. M.: Circulation of Heavy Ions and Their Dynamical Effects in the Magnetosphere: Recent Observations and Models, Space Sci. Rev., 184, 173-235, https://doi.org/10.1007/s11214-014-0104-0, 2014.

Kronberg, E. A., Grigorenko, E. E., Haaland, S. E., Daly, P. W., Kistler, L. M., and Dandouras, I.: Distributions of energetic oxygen and hydrodgen in the near Earth plasma sheet, J. Geophys. Res., 120, 3415-3431, https://doi.org/10.1002/2014JA020882, 2015.

Kronberg, E. A., Grigorenko, E. E., Turner, D. L., Daly, P. W., Khotyaintsev, Y., and Kozak, L.: Comparing and contrasting dispersionless injections at geosynchronous orbit during a substorm event, J. Geophys. Res., 122, 3055-3072, https://doi.org/10.1002/2016JA023551 2017.

Lanzerotti, L. J., Roberts, C. S., Brown, W. L.: Temporal variations in the electron flux at synhronous altitudes, J. Geophys. Res., 72, 5883-5902, https://doi.org/10.1029/JZ072i023p05893, 1967.

Le Contel, O., Roux, A., Jacquey, C., Robert, P., Berthomier, M., Chust, T., Grison, B., Angelopoulos, V., Sibeck, D., Chaston, C. C., Cully, C. M., Ergun, B., Glassmeier, K.-H., Auster, U., McFadden, J., Carlson, C., Larson, D., Bonnell, J. W., Mende, S., Russell, C. T., Donovan, E., Mann, I., and Singer, H.: Quasi-parallel whistler mode waves observed by THEMIS during near-earth dipolarizations, Ann. Geophys., 27, 2259-2275, https://doi.org/10.5194/angeo-27-2259-2009, 2009.

Li, X., Sarris, T. E., Baker, D. N., Peterson, W. K., and Singer, H. J.: Simulation of energetic particle injections associated with a substorm on August 27, 2001, Geophys. Res. Lett., 30, 1004, https://doi.org/10.1029/2002GL015967, 2003.

Liu, J., Angelopoulos, V., Runov, A., and Zhou, X.-Z.: On the current sheets surrounding dipolarizing flux bundles in the magnetotail: The case for wedgelets, J. Geophys. Res., 118, 2000-2020, https://doi.org/10.1002/jgra.50092, 2013.
Lui, A. T. Y.: Current disruption in the Earth's magnetosphere: Observations and models, J. Geophys. Res., 101, 13067-13088, 1996.

Lui, A. T. Y.: Reduction of the cross-tail current during near-Earth dipolarization with multisatellite observations, J. Geophys. Res., 116, A12239, https://doi.org/10.1029/2011JA017107, 2011.

Lui, A. T. Y., Yoon, P. H., Mok, C., and Ryu, C.-M.: Inverse cascade feature in current disruption, J. Geophys. Res., 113, A00C06, https://doi.org/10.1029/2008JA013521, 2008.

Luo, H., Kronberg, E. A., Grigorenko, E. E., Fränz, M., Daly, P. W., Chen, G. X., Du, A. M., Kistler, L. M., and Wei, Y.: Evidence of strong energetic ion acceleration in the near-Earth magnetotail, Geophys. Res. Lett., 41, 3724-3730, https://doi.org/10.1002/2014GL060252, 2014

Lutsenko, V. N., Kudela,, K., and Sarris, E. T.: The DOK-2 experiment to study energetic particles by the Tail probe and Auroral probe satellites in the INTERBALL project, Cosmic Res., 36, 93-102, 1998.

Mauk, B. H. and McIlwain, C. E.: Correlation of Kpwith the substorm injected plasma boundary, J. Gephys. Res., 79, 22, 1974.

McFadden, J. P., Carlson, C. W., Larson, D., Angelopoulos, V., Ludlam, M., Abiad, R., Elliott, B., Turin, P., and Marckwordt, M.: The THEMIS ESA plasma instrument and in-flight calibration, Space Sci. Rev., 141, 277-302, https://doi.org/10.1007/s11214008-9440-2, 2008a.

McIlwain, C. E.: Substorm injection boundaries, in: Magnetospheric Physics, edited by: McComas, B. M., 143 pp., D. Reidel, Hingham, Mass, 1974.

McPherron, R. L., Russell, C. T., and Aubry, M. A.: Satellite studies of magnetospheric substorms on august $15,1968,9$, phenomenological model for substorms, J. Geophys. Res., 78, 3131-3149, 1973.

Moore, T. E., Arnoldy, L., Feynman, J., and Hardy, D. A.: Propagating substorm injection fronts, J. Geophys. Res., 86, 6713-6726, https://doi.org/10.1029/JA086iA08p06713, 1981.

Nakamura, R., Baumjohann, W., Klecker, B., Bogdanova, Y.,Balogh, A., Reme, H., Bosqued, J. M., Dandouras, I., Sauvaud, J. A., Glassmeier, K. H., Kistler, L., Mouikis, C., Zhang, T. L., Eichelberger, H., and Runov, A.: Motion of the dipolarization front during a flow burst event observed by Cluster, Geophys. Res. Lett., 29, 1942, https://doi.org/10.1029/2002GL015763, 2002.

Nakamura, R., Baumjohann, W., Mouikis, C., Kistler, L. M., Runov, A., Volwerk, M., Asano, Y., Voros, Z., Zhang, T. L., Klecker, B., Reme, H., and Balogh, A.: Spatial scale of high-speed flows in the plasma sheet observed by Cluster, Geophys. Res. Lett., 31, L09804, https://doi.org/10.1029/2004GL019558, 2004.

Nakamura, R., Retinò, A., Baumjohann, W., Volwerk, M., Erkaev, N., Klecker, B., Lucek, E. A., Dandouras, I., André, M., and Khotyaintsev, Y.: Evolution of dipolarization in the nearEarth current sheet induced by Earthward rapid flux transport, Ann. Geophys., 27, 1743-1754, https://doi.org/10.5194/angeo27-1743-2009, 2009.

Øieroset, M., Lin, R. P., Phan, T. D., Larson, D. E., and Bale, S. D.: Evidence for electron acceleration up to $300 \mathrm{keV}$ in the magnetic reconnection diffusion region in the earth's magnetotail, Phys. Rev. Lett., 89, 195001, https://doi.org/10.1103/PhysRevLett.89.195001, 2002. 
Pan, Q., Ashour-Abdalla, M., El-Alaoui, M., Walker, R. J., and Goldstein, M. L.: Adiabatic acceleration of suprathermal electrons associated with dipolarization fronts, J. Geophys. Res., 117, A12224, https://doi.org/10.1029/2012JA018156, 2012.

Panov, E. V., Nakamura, R., Baumjohann, W., Angelopoulos, V., Petrukovich, A. A., Retino, A., Volwerk, M., Takada, T., Glassmeier, K. H., McFadden, J. P., and Larson, D.: Multiple overshoot and rebound of a bursty bulk flow, Geophys. Res. Lett., 37, L08103, https://doi.org/10.1029/2009GL041971, 2010.

Panov, E. V., Artemyev, A. V., Baumjohann, W., Nakamura, R., and Angelopoulos, V.: Transient electron precipitation during oscillatory BBF braking: THEMIS observations and theoretical estimates. J. Geophys. Res., 118, 3065-3076, https://doi.org/10.1002/jgra.50203, 2013.

Panov, E. V., Wolf, R. A., Kubyshkina, M. V., Nakamura, R., and Baumjohann, W.: Anharmonic oscillatory flow braking in the Earth's magnetotail, Geophys. Res. Lett., 42, 3700-3706, https://doi.org/10.1002/2015GL064057, 2015.

Paschmann, G. and Daly, P. W.: Analysis Methods for MultiSpacecraft Data, The International Space Science Institute Hallerstrasse 6, CH-3012 Bern, Switzerland, 1998.

Roux, A., Contel Le, C. O., Bouabdellah, C. A., de la Porte, B., Alison, D., Ruocco, S., and Vassal, M. C.: The Search Coil Magnetometer for THEMIS, Space Sci. Rev., 141, 265-275, https://doi.org/10.1007/s11214-008-9455-8, 2008.

Runov, A., Angelopoulos, V., Sitnov, M. I., Sergeev, V. A., Bonnell, J., McFadden, J. P., Larson, D., Glassmeier, K.H., and Auster, U.: THEMIS observations of an earthwardpropagating dipolarization front, Geophys. Res. Lett., 36, L14106, https://doi.org/10.1029/2009GL038980, 2009.

Runov, A., Angelopoulos, V., Zhou, X.-Z., Zhang, X.-J., Plaschke, S. U. F., and Bonnell, J.: A THEMIS multicase study of dipolarization fronts in the magnetotail plasma sheet, J. Geophys. Res., 116, A05216, https://doi.org/10.1029/2010JA016316, 2011.

Runov, A., Sergeev, V. A., Angelopoulos, V., Glassmeier, K.H., and Singer, H. J.: Diamagnetic oscillations ahead of stopped dipolarization fronts, J. Geophys. Res., 119, 1643-1657, https://doi.org/10.1002/2013JA019384, 2014.

Runov, A., Angelopoulos, V., Gabrielse, C., Liu, J., Turner, D. L., and Zhou, X.-Z., Average thermodynamic and spectral properties of plasma in and around dipolarizing flux bundles, J. Geophys. Res., 120, 4369-4383 https://doi.org/10.1002/2015JA021166, 2015.

Sarris, E. T., Krimigis, S. M., and Armstrong, T. P.: Observations of magnetospheric bursts of high-energy protons and electrons at 35 with $\operatorname{Imp~7,~J.~Geophys.~Res.,~81,~2341-2355,~}$ https://doi.org/10.1029/JA081i013p02341, 1976.

Sarris, T. E., Tsaggas, X. U. N., and Paschalidis, N.: Modeling energetic particle injections in dynamic pulse fields with varying propagation speeds, J. Geophys. Res., 107, 1033-1043, https://doi.org/10.1029/2001JA900166, 2002.

Schmid, D., Volwerk, M., Nakamura, R., Baumjohann, W., and Heyn, M.: A statistical and event study of magnetotail dipolarization fronts, Ann. Geophys., 29, 1537-1547, https://doi.org/10.5194/angeo-29-1537-2011, 2011.

Sergeev, V. A., Yahnin, D. A., Liou, K., Thomsen, M. F., and Reeves, G. D.: Narrow plasma streams as a candidate to populate the inner magnetosphere, in: The Inner Magnetosphere Physics Modeling, Gephys. Monogr. Ser., edited by: Pulkkinen, T. I.,
Tsyganenko, N. A., and Friedel, R. H. W., p. 155, AGU, Washington, D.C., https://doi.org/10.1029/155GM07, 2005.

Sergeev, V., Angelopoulos, V., Apatenkov, S., Bonnell, J., Ergun, R., Nakamura, R., McFadden, J., Larson, D., and Runov, A.: Kinetic structure of the sharp injection/dipolarization front in the flow-braking region, Geophys. Res. Lett., 36, L21105, https://doi.org/10.1029/2009GL040658, 2009.

Sergeev, V. A., Tsyganenko, N. A., Smirnov, M. V., Nikolaev, A. V., Singer, H. J., and Baumjohann, W.: Magnetic effects of the substorm current wedge in a "spreadout wire model" and their comparison with ground, geosynchronous, and tail lobe data, J. Geophys. Res., 116, A07218, https://doi.org/10.1029/2011JA016471, 2011.

Sergeev, V. A., Angelopoulos, V., and Nakamura, R.: Recent advances in understanding substorm dynamics, Geophys. Res. Lett., 39, L05101, https://doi.org/10.1029/2012GL050859, 2012.

Shiokawa, K., Baumjohann, W., and Haerendel, G.: Braking of high-speed flows in the near-Earth tail, Geophys. Res. Lett., 24, 1179-1182, 1997.

Shiokawa, K., Miyashita, Y., Sinohara, I., and Matsuoka, A.: Decrease in BZ prior to the dipolarization in the near-Earth plasma sheet, J. Geophys. Res., 110, A09219, https://doi.org/10.1029/2005JA011144, 2005.

Stawarz, J. E., Ergun, R. E., and Goodrich, K. A.: Generation of high-frequency electric field activity by turbulence in the Earth's magnetotail, J. Geophys. Res., 120, 1845-1866, https://doi.org/10.1002/2014JA020166, 2015.

SuperMAG: Observations of the global magnetic field, available at: http://supermag.jhuapl.edu/, last access: 13 February, 2018.

THEMIS:Time History of Events and Macroscale Interactions during Substorms, available at: http://themis.ssl.berkeley.edu/index. shtml, last acces: 4 March, 2018.

Ukhorskiy, A. Y., Sitnov, M. I., Merkin, V. G., and Artemyev, A. V.: Rapid acceleration of protons upstream of earthward propagating dipolarization fronts, J. Geophys. Res.-Space Phys., 118, 49524962, https://doi.org/10.1002/jgra.50452, 2013.

Vilberg, H., Khotyaintsev, Yu. V., Vaivads, A., André, M., Fu, H. S., and Cornilleau-Wehrlin, N.: Whistler mode waves at magnetotail dipolarization fronts, J. Geophys. Res., 119, 2605-2611, https://doi.org/10.1002/2014JA019892, 2014.

Wilken, B., Daly, P. W., Mall, U., Aarsnes, K., Baker, D. N., Belian, R. D., Blake, J. B., Borg, H., Büchner, J., Carter, M., Fennell, J. F., Friedel, R., Fritz, T. A., Gliem, F., Grande, M., Kecskemety, K., Kettmann, G., Korth, A., Livi, S., McKenna-Lawlor, S., Mursula, K., Nikutowski, B., Perry, C. H., Pu, Z. Y., Roeder, J., Reeves, G. D., Sarris, E. T., Sandahl, I., Söraas, F., Woch, J., and Zong, Q.-G.: First results from the RAPID imaging energetic particle spectrometer on board Cluster, Ann. Geophys., 19, 1355-1366, https://doi.org/10.5194/angeo-19-1355-2001, 2001.

Yao, Z. H., Pu, Z. Y., Fu, S. Y., Angelopoulos, V., Kubyshkina, M., Xing, X., Lyons, L., Nishimura, Y., Xie, L., Wang, X. G., Xiao, C. J., Cao, X., Liu, J., Zhang, H., Nowada, M., Zong, Q. G., Guo, R. L., Zhong, J., and Li, J. X.: Mechanism of substorm current wedge formation: THEMIS observations, Geophys. Res. Lett., 39, L13102, https://doi.org/10.1029/2012GL052055, 2012.

Zaharia, S., Cheng, C. Z., and Johnson, J. R.: Particle transport and energization in association with substorms, J. Geophys. Res., 105, 18741-18752, https://doi.org/10.1029/1999JA000407, 2000 . 
Zhang, X. and Angelopoulos, V.: On the relationship of electrostatic cyclotron harmonic emissions with electron injections and dipolarization fronts, J. Geophys. Res., 119, 2536-2549, https://doi.org/10.1002/2013JA019540, 2014.

Zhou, M., Ashour-Abdalla, M., Deng, X., Schriver, D., ElAlaoui, M., and Pang, Y.: THEMIS observation of multiple dipolarization fronts and associated wave characteristics in the near-Earth magnetotail, Geophys. Res. Lett., 36, L20107, https://doi.org/10.1029/2009GL040663, 2009.
Zhou, M., Ashour-Abdalla, M., Deng, X. H., El-Alaoui, M., Richard, R. L., and Walker, R. J.: Modeling substorm ion injection observed by the THEMIS and LANL spacecraft in the near-Earth magnetotail, J. Geophys. Res., 116 A08222, https://doi.org/10.1029/2010JA016391, 2011.

Zhou, X., Angelopoulos, V., Sergeev, V. A., and Runov, A.: Accelerated ions ahead of earthward propagating dipolarization fronts, J. Geophys. Res., 115, A00I03, https://doi.org/10.1029/2010JA015481, 2010. 